\title{
Tuning the Structures of Metal Organic Frameworks via a Mixed-Linker Strategy for Ethylene/Ethane Kinetic Separation
}

Richelle Lyndon ${ }^{\dagger}$, Wenqin You ${ }^{\dagger}$, Yao Ma ${ }^{\dagger}$, John Bacsa ${ }^{\ddagger}$, Yutao Gong ${ }^{\dagger}$, Eric Stangland ${ }^{\S}$, Krista S. Walton ${ }^{\dagger}$, David S. Sholl ${ }^{\dagger}$, Ryan P. Lively* ${ }^{\dagger}$

†School of Chemical \& Biomolecular Engineering, Georgia Institute of Technology, 311 Ferst Drive NW, Atlanta, Georgia 30332, United States

$\ddagger$ School of Chemistry \& Biochemistry, Georgia Institute of Technology, 901 Atlantic Drive, Atlanta, Georgia 30332, United States

§Corporate Research \& Development, The Dow Chemical Company, Midland, Michigan 48674, United States

Corresponding author. E-mail: ryan.lively@chbe.gatech.edu.

Table of Contents:

\section{Supporting Information}

Section 1: Synthesis Details

Section 2: Materials Characterization

Section 3: Single crystal X-ray crystallography

Section 4: Tabular Data 


\section{Section 1: Synthesis Details}

\subsection{Materials}

All reagents were used without further purification. Zinc acetate dihydrate $\left(\mathrm{Zn}(\mathrm{OAc})_{2},>97 \%\right)$, benzimidazole (BIM, 99\%), dimethylformamide (DMF, >99.8\%) were purchased from Alfa Aesar. Benzotriazole (BTA, 99\%) and zinc bromide dihydrate were purchased from Sigma Aldrich. Ethanol (200 proof) was obtained from Koptec, and methanol (99.8\%) and ammonium hydroxide (28-30\%) were obtained from BDH Chemicals.

Nitrogen (UHP), carbon dioxide (99.999\%), methane (UHP), ethane (99.99\%), and helium (UHP) were purchased from Airgas, and Ethylene (99.99\%) was purchased from Matheson.

\subsection{Synthesis of GT-18}

The synthesis method was modified from a previously reported procedure for the synthesis of triazole-based MOFs. ${ }^{1}$ Zinc acetate dihydrate $(0.805 \mathrm{~g}, 3.7 \mathrm{mmol})$ was first dissolved in a mixed solvent of DMF, ethanol, DI water and ammonium hydroxide (30\%) (20 mL, 5:5:7.5:2.5 v/v). BTA $(1.029 \mathrm{~g}, 8.6 \mathrm{mmol})$ and BIM $(0.256 \mathrm{~g}, 2.2 \mathrm{mmol})$ are slowly added to the zinc solution while stirring. The reaction mixture was then left stirring at $150 \mathrm{rpm}$ for 4 days at room temperature. The white powder was obtained after centrifuge, rinsed with DMF $(\sim 20 \mathrm{~mL})$ and washed with methanol $(3 \mathrm{x} \sim 20 \mathrm{~mL})$. The powder was then soaked in methanol and replaced with fresh methanol up to 3 times a day for 2 days, and then dried and activated at $200{ }^{\circ} \mathrm{C}$ under vacuum for at least 12 hours prior to sorption measurement.

${ }^{1} \mathrm{H}$ NMR (400 MHz, DMSO- $\left.d_{6} / \mathrm{DCl} / \mathrm{D} 2 \mathrm{O}\right) \delta 9.57(\mathrm{~s}, 1 \mathrm{H}), 7.87-7.82(\mathrm{~m}, 2 \mathrm{H}), 7.81(\mathrm{dd}, J=6.2$, $3.1 \mathrm{~Hz}, 2 \mathrm{H}), 7.53(\mathrm{dd}, J=6.2,3.2 \mathrm{~Hz}, 2 \mathrm{H}), 7.40(\mathrm{dd}, J=6.3,3.1 \mathrm{~Hz}, 2 \mathrm{H})$.

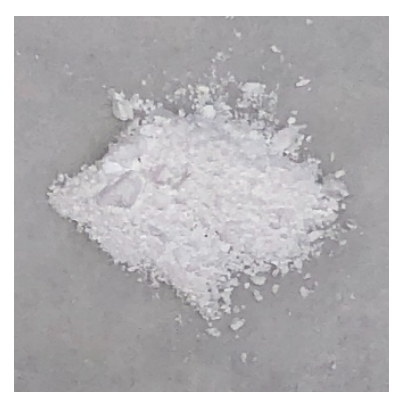

\subsection{Synthesis of ZIF-7 $M_{20}$}

The synthesis method was modified from a previously reported procedure for the synthesis of triazole-based MOFs. ${ }^{1}$ Zinc acetate dihydrate $(0.805 \mathrm{~g}, 3.7 \mathrm{mmol})$ was first dissolved in a mixed solvent of DMF, ethanol, DI water and ammonium hydroxide (30\%) (20 mL, 5:5:7.5:2.5 v/v). BTA $(0.645 \mathrm{~g}, 5.4 \mathrm{mmol})$ and BIM $(0.638 \mathrm{~g}, 5.4 \mathrm{mmol})$ are slowly added to the zinc solution while stirring. The reaction mixture was then left stirring at $150 \mathrm{rpm}$ for 4 days at room temperature. The white powder was obtained after centrifuge, rinsed with DMF $(\sim 20 \mathrm{~mL})$ and washed with methanol $(3 \mathrm{x} \sim 20 \mathrm{~mL})$. The powder was then soaked in methanol and replaced with fresh methanol up to 3 times a day for 2 days, and then dried and activated at $150{ }^{\circ} \mathrm{C}$ under vacuum for at least 12 hours prior to sorption measurement. 


\subsection{Synthesis of ZIF-7 $M_{46}$}

The synthesis method was modified from a previously reported procedure for the synthesis of triazole-based MOFs. ${ }^{1}$ Zinc acetate dihydrate $(0.808 \mathrm{~g}, 3.7 \mathrm{mmol})$ was first dissolved in a mixed solvent of DMF, ethanol, DI water and ammonium hydroxide $(30 \%)(20 \mathrm{~mL}, 5: 5: 7.5: 2.5 \mathrm{v} / \mathrm{v})$. BTA $(0.907 \mathrm{~g}, 7.6 \mathrm{mmol})$ and $\operatorname{BIM}(0.383,3.2 \mathrm{mmol})$ are slowly added to the zinc solution while stirring. The reaction mixture was then left stirring at $150 \mathrm{rpm}$ for 4 days at room temperature. The white powder was obtained after centrifuge, rinsed with DMF $(\sim 20 \mathrm{~mL})$ and washed with methanol $(3 \mathrm{x} \sim 20 \mathrm{~mL})$. The powder was then soaked in methanol and replaced with fresh methanol up to 3 times a day for 2 days, and then dried and activated at $150{ }^{\circ} \mathrm{C}$ under vacuum for at least 12 hours prior to sorption measurement.

\subsection{Synthesis of $\left[\mathrm{Zn}(\mathrm{BTA})_{2}\right]_{n}$}

The synthesis method was modified from a previously reported procedure for the synthesis of triazole-based MOFs. ${ }^{1}$ Zinc acetate dihydrate $(1.611 \mathrm{~g}, 7.3 \mathrm{mmol})$ was first dissolved in a mixed solvent of DMF, ethanol, DI water and ammonium hydroxide $(30 \%)(40 \mathrm{~mL}, 5: 5: 7.5: 2.5 \mathrm{v} / \mathrm{v})$. BTA $(2.572 \mathrm{~g}, 21.6 \mathrm{mmol})$ are slowly added to the zinc solution while stirring. The reaction mixture was then left stirring at $150 \mathrm{rpm}$ for 4 days at room temperature. The white powder was obtained after centrifuge, rinsed with DMF $(\sim 20 \mathrm{~mL})$ and washed with methanol $(3 \mathrm{x} \sim 20 \mathrm{~mL})$. The powder was then soaked in methanol and replaced with fresh methanol up to 3 times a day for 2 days, and then dried and activated at $150{ }^{\circ} \mathrm{C}$ under vacuum for at least 12 hours prior to sorption measurement.

\subsection{Synthesis of Larger GT-18 Single Crystals ( $\sim 50 \mu \mathrm{m})$}

Zinc acetate dihydrate $(0.323 \mathrm{~g}, 1.5 \mathrm{mmol})$ was first dissolved in a mixed solvent of DMF, ethanol, DI water and ammonium hydroxide (30\%) (8 mL, 2:2:3.5:1 v/v). BTA $(0.206 \mathrm{~g}, 1.7$ $\mathrm{mmol})$ and BIM $(0.052 \mathrm{~g}, 0.4 \mathrm{mmol})$ are added to the zinc solution. The vial was shaken briefly and then the mixture was left to react for 6 days at room temperature. The single crystals were washed with DMF and submitted for analysis.

\subsection{Synthesis of ZIF-7}

ZIF-7 was synthesized according to previous literature. ${ }^{2}$ Zinc bromide dihydrate (3.571 g, 15.9 $\mathrm{mmol})$ and BIM (2.489 g, $21.1 \mathrm{mmol})$ were dissolved to $240 \mathrm{~mL}$ of DMF. DEA (3.762 $\mathrm{g}$ ) was then added to to the mixture. The synthesis solution was sealed in autoclaves and rotated at $40 \mathrm{rpm}$ at $130{ }^{\circ} \mathrm{C}$ for 1 day. The resulting white powder was collected by filtration, washed with DMF, and then with methanol. The ZIF-7 crystals were soaked in methanol at $60{ }^{\circ} \mathrm{C}$. The solvent was replaced with fresh methanol twice per day up to 3 days and dried and activated at $150{ }^{\circ} \mathrm{C}$ under vacuum for at least 12 hours prior to sorption measurement.

\subsection{Preparation of ZIF-7-M by Postsynthetic Ligand Exchange}

ZIF-7M-PSE was prepared according to a previously reported method. ${ }^{3}$ Methanol solution with benzotriazole concentration of $\sim 1-2 \mathrm{mg} / \mathrm{mL}$ was placed in a $50 \mathrm{~mL}$ centrifuge tube $\sim 200 \mathrm{mg}$ of ZIF-7 were immersed in the solution and placed in a water bath at $30{ }^{\circ} \mathrm{C}$ for several days. The solution was replaced daily with a fresh solution of the same concentration of benzotriazole. The white solid was dried and activated at $150{ }^{\circ} \mathrm{C}$ under vacuum for at least 12 hours prior to sorption measurement prior to sorption measurements. 


\section{Section 2: Materials Characterization}

\subsection{Powder X-Ray Diffraction (PXRD) Measurements}

PXRD patterns of samples were collected from a PANalytical X'Pert PRO Alpha-1 at $40 \mathrm{kV}$ and $40 \mathrm{~mA}$ with $\mathrm{Cu}-\mathrm{K} \alpha$ radiation of $1.54184 \AA$. The patterns were collected with a step size of $0.017^{\circ}$ $2 \theta$ and scan time of $12 \mathrm{~s} /$ step over $2 \theta$ range of $5-50^{\circ}$.

\subsection{Nuclear Magnetic Resonance Spectroscopy (NMR)}

${ }^{1} \mathrm{H}$ NMR spectra were obtained on a Bruker AVANCE III 400 NMR. GT-18 was digested and dissolved by sonication in $100 \mu \mathrm{l}$ of DCl/D2O (35 wt\%) solution in $600 \mu \mathrm{l}$ DMSO-d6. DMSO-d6 was used as a reference peak. Due to some peak overlap, accurate integration is not possible. Thus, the relative ratio of linkers of MOF samples was determined by digesting and dissolving the MOF in only DCl/D2O solution, and the sample was locked to D2O. Synthesis of GT-18 was repeated two more times (including the synthesis of larger crystals) to ensure the reproducibility and the BTA content are consistent, ranging from $68-71 \%$.

\subsection{Scanning Electron Microscopy (SEM)}

Scanning Electron Microscopy (SEM) images were obtained using a Hitachi SU8230 scanning electron microscope with an accelerating voltage of 1-2 kV and emission current of $5 \mu \mathrm{A}$ at a working distance of $4-8 \mathrm{~mm}$. The samples were prepared by dispersing the material onto doublesided conductive copper tape attached to a flat aluminum sample stub.

\subsection{Fluorescence Confocal Laser Scanning Microscopy (CLSM)}

Confocal microscopy was performed on a Zeiss LSM 780/PS.1 super-resolution microscope equipped with a standard photomultiplier tube, 32-channel spectral GaAsP detector, a cooled GaAsP detector. The objective used is a 100X / 1.46 (oil) TIRF $\alpha$-Plan Apochromat (wd = $0.110 \mathrm{~mm})$. Samples were placed on cell imaging dishes with coverglass bottom $(170 \mu \mathrm{m}, 35 \mathrm{x} 10$ $\mathrm{mm}$, Eppendorf) and coated with immersion oil (Immersol ${ }^{\mathrm{TM}} 518 \mathrm{~F}$, Zeiss). Spectral data were acquired under the lambda-mode and a $405 \mathrm{~nm}$ excitation laser was used $(30 \mathrm{~mW})$. Jayachandrababu et al. have shown that CLSM can be used to obtain information on the linker distribution of MOF crystals containing mixed linkers. ${ }^{4}$ Linear unmixing analyses on MOF samples were conducted to determine the relative contribution from each linker throughout the MOF crystals. For the unmixing experiment, free BIM and BTA linkers (uncoordinated) emission spectra were used as reference spectra. Blue and green colors were assigned to BTA and BIM, respectively. Z-stack of the MOF images were acquired to look at the localization of each linker throughout the crystal.

\subsection{Thermal Gravimetric Analysis (TGA)}

Thermogravimetric analyses were recorded on a TGA Q500 (TA Instruments) with a heating rate of $5{ }^{\circ} \mathrm{C} / \mathrm{min}$ to $700{ }^{\circ} \mathrm{C}$ under nitrogen flow. The samples were placed in clean platinum sample pans.

\subsection{Vapor Sorption Measurement}

A single component water vapor sorption isotherm of activated GT-18 was measured with a VTI$\mathrm{SA}+$ (TA Instruments). The sample was activated at $200{ }^{\circ} \mathrm{C}$ under vacuum overnight and then dried in situ at $115^{\circ} \mathrm{C}$ for approximately $8 \mathrm{~h}$ under flowing nitrogen. The isotherm was collected 
within a range of $0-95 \% \mathrm{RH}$ at $25^{\circ} \mathrm{C}$. The equilibrium criteria for each step was set for mass changes less than $0.001 \mathrm{wt} \%$ in a 5 min period.

\subsection{Fourier-Transform Infrared Spectroscopy (FTIR)}

Infrared spectra were collected using a Thermo Scientific Nicolet iS10 FT-IR spectrometer using the ATR mode with a resolution of $2 \mathrm{~cm}^{-1}$ and 32 accumulated scans.

\subsection{Density Functional Theory (DFT) and Binding Energy Calculations}

We calculate binding affinities with spatially periodic DFT using the Vienna Ab Initio Simulation Package (VASP), ${ }^{5}$ along with a plane-wave basis set and projected-augmented wave (PAW) ${ }^{6}$ pseudopotentials. All calculations used the Perdew, Burke, and Ernzerhof (PBE) ${ }^{7}$ GGA XC functional with D3 dispersion corrections (PBE-D3) ${ }^{8}$. During geometry relaxation we simultaneously optimized both the cell volume and ionic positions using a plane-wave cutoff energy of $520 \mathrm{eV}$ and gamma-point sampling for Brillouin zone integration. Using a quasi-Newton method, we relaxed geometries until the force on each atom is smaller than $0.03 \mathrm{eV} / \AA$.

Initial coordinates of GT-18 were taken from single crystal structure containing solvent (DMF). GT-18 was first energy minimized with the solvent. Then the solvent was removed manually from the structure and the GT-18 energy was minimized again. After the geometry optimization, the optimized lattice parameters of GT-18 with and without solvent were obtained and compared with experimental structure with DMF solvent.

The binding energies of adsorbed DMF solvent molecules were defined by

$$
-\Delta E=E_{\mathrm{DMF}+M O F}-E_{M O F}-E_{D M F},
$$

where $E_{D M F+M O F}, E_{M O F}$, and $E_{D M F}$ represent the energy of a MOF with an adsorbed DMF molecule, the energy of the empty MOF, and the energy of the adsorbate (DMF molecule) in the gas phase, respectively. With this convention, $\Delta E$ is positive when adsorption is exothermic.

\subsection{Gas Adsorption Measurements}

Single component equilibrium gas adsorption isotherms data were collected on an ASAP 2020 (Micromeritics) from 0-1 bar at $298 \mathrm{~K}$ using an equilibration interval of 90 seconds. Approximately 100-150 mg of GT-18 and other ZIF samples were used for measurements. ZIF samples (ZIF-7, ZIF-7M-PSE, ZIF-7M ${ }_{\mathrm{x}}$ ) were activated at $150{ }^{\circ} \mathrm{C}$ under dynamic vacuum for at least 12 hours, as reported in the literature for ZIF-7 activation. ${ }^{9}$ GT-18 was activated at higher temperature $\left(200^{\circ} \mathrm{C}\right)$ under dynamic vacuum for at least 12 hours to remove sorbed gas molecules (lower gas sorption uptakes observed at $150{ }^{\circ} \mathrm{C}$ activation). Due to the slow kinetic diffusion of ethane and ethylene through the narrow pores, GT-18 samples were re-activated under dynamic vacuum at $200{ }^{\circ} \mathrm{C}$ for 2 days.

\subsection{Molecular Simulations of Adsorption}

Grand Canonical Monte Carlo (GCMC) methods as implemented in RASPA ${ }^{10}$ were used to investigate the single-component adsorption of ethylene and ethane in GT-18. These simulations used the solvent-free DFT optimized structure described above, with atomic point charges assigned from our DFT calculations by the Density Derived Electrostatic and Chemical (DDEC) charge assignment method. ${ }^{11}$ GT-18 was treated as rigid for all GCMC simulations. Nonbonded 
Van der Waals interactions between adsorbates and the framework atoms were calculated using a Lennard-Jones $(\mathrm{L}-\mathrm{J})$ potential truncated at a $12 \AA$ cutoff. $\mathrm{L}-\mathrm{J}$ parameters were taken from the DREIDING ${ }^{12}$ force field for the framework atoms and the TraPPE ${ }^{13-14}$ force field for adsorbates. The parameters for the interactions between framework atoms and adsorbates were obtained by Lorentz-Berthelot (LB) mixing rules. Nonbonded electrostatic interaction was calculated by Ewald summation method. ${ }^{15}$ Simulations were performed using a simulation volume containing $2 * 2 * 2$ unit cells. For each GCMC simulation, after $1 \times 10^{4}$ initialization cycles for equilibration, a production run was followed with $1 \times 10^{5}$ cycles; tests indicated this was sufficient to get converged results.

\subsection{Kinetic Uptake Measurements}

Single-component diffusion and excess uptake of ethane and ethylene were measured in an HPVAII (Micromeritics) at $298 \mathrm{~K}$ with equilibrium criteria of $0.03 \mathrm{mbar}$ per min for at least $10 \mathrm{~min}$ for ethane and $20 \mathrm{~min}$ for ethylene to avoid premature data collection. A small amount of sample (approximately $0.040 \mathrm{~g}$ ) was used to minimize intra-particle resistance.

The Fickian transport diffusion coefficient of ethylene and ethane in GT-18 can be determined by the volumetric sorption experiment. The obtained uptake change data in isotherms are first normalized using ${ }^{16}$

$$
\frac{M_{t}}{M_{\infty}}=\frac{M(t)-M_{0}}{M(t=\infty)-M_{0}}
$$

where $M(t)$ is the amount of ethylene or ethane into the GT-18 at time point $t$ and $M(t=\infty)$ is the theoretical sorption amount after infinite time.

Generally, the experimental kinetic uptake curve can be fitted by a Fickian mass transfer model to determine the transport diffusion coefficient. Based on the crystallographic data, GT-18 possesses a 1D pore channel along the $a$-axis, which corresponds to the longest dimension of the rod-shaped crystals. Therefore, guest molecules can only enter the particle through the direction of the particle length. Therefore, the kinetic mass uptake processes can be described by the 1D Fickian diffusion model. For a cylindrical particle subject to a step change in adsorbate concentration at its external surface at time zero, the mathematical infinite series solution for the Fickian uptake is given by ${ }^{16}$

$$
\left.\frac{M_{t}}{M_{\infty}}\right|_{L_{i}}=1-\sum_{n=0}^{\infty} \frac{8}{(2 n+1)^{2} \pi^{2}} \exp \left[-\frac{D(2 n+1)^{2} \pi^{2} t}{4 L_{i}^{2}}\right]
$$

where $t$ is the diffusion time, $L_{i}$ is the half-length of the MOF particle $i$, and $D$ is the transport diffusivity. 
The apparent Fickian uptake considering particle size distribution can be approximated as the superposition of the solutions for each particle within the population ${ }^{17}$ :

$$
\left.\frac{M_{t}}{M_{\infty}}\right|_{P S D}=\left.\sum_{i} X_{i} \frac{M_{t}}{M_{\infty}}\right|_{L_{i}}
$$

where $X_{i}$ is the volume-weighted fraction of particles with a half-length of $L_{i}$ and can be obtained from $X_{i}=V_{i} / \sum_{i} V_{i} . V_{i}$ is the volume of particles of a half-length $L_{i}$.

\subsection{Dynamic Column Breakthrough Experiment}

GT-18 powder $(0.2578 \mathrm{~g})$ was packed into a $1 / 4$ "' Swagelok tube with bed length of 2 " and activated under a helium flow rate of $10 \mathrm{cc} / \mathrm{min}$ at $200{ }^{\circ} \mathrm{C}$ for $24 \mathrm{~h}$. The breakthrough curves were then obtained by switching the helium flow to a flow containing equimolar ethylene/ethane mixture at a total flow rate of $5 \mathrm{cc} / \mathrm{min}$ at $20{ }^{\circ} \mathrm{C}$. The concentration of ethylene and ethane at the outlet was monitored with an Isochema Quadrupole Mass Spectrometer (Hiden). When the breakthrough curves appeared, the valves before and after the bed, and the ethylene/ethane gas mixture were closed at the same time to start the batch adsorption experiment on GT-18. After $2.5 \mathrm{~h}$, the valves were opened to flow helium gas at a flow rate of $10 \mathrm{cc} / \mathrm{min}$ and carry the rest ethylene/ethane to the mass spectrometry. The purity profile of ethylene after batch adsorption was calculated through the concentration change of ethylene and ethane. 

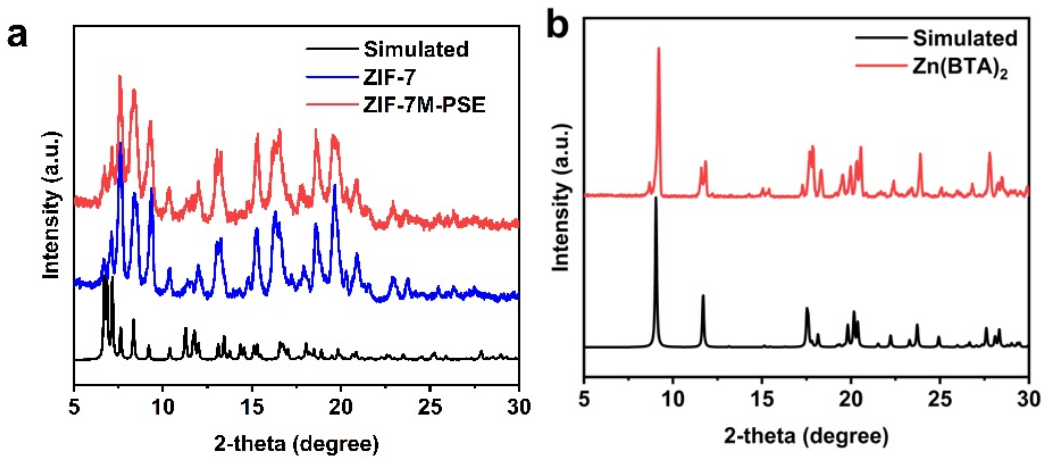

Figure S1. PXRD patterns of the control samples. (a) ZIF-7M-PSE prepared through a postsynthetic ligand exchange method showed no changes in the ZIF-7 framework and the pattern correspond well with the simulated PXRD pattern of an activated ZIF-7 phase. (b) PXRD patterns of $\left[\mathrm{Zn}(\mathrm{BTA})_{2}\right]_{n}$ obtained from using a similar synthesis condition as GT-18 in the absence of the BIM linker.

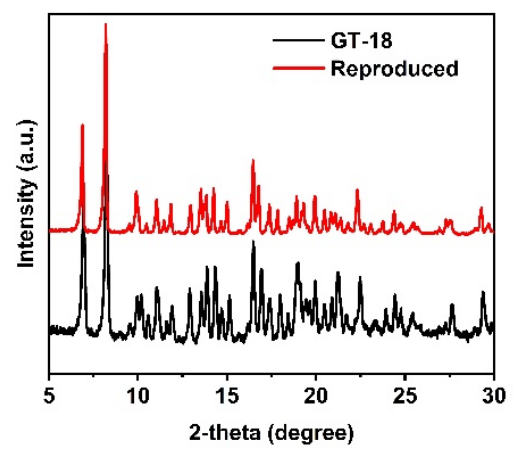

Figure S2. PXRD patterns of the reference and reproduced GT-18 samples. The synthesis was reproduced using only information from the manuscript.

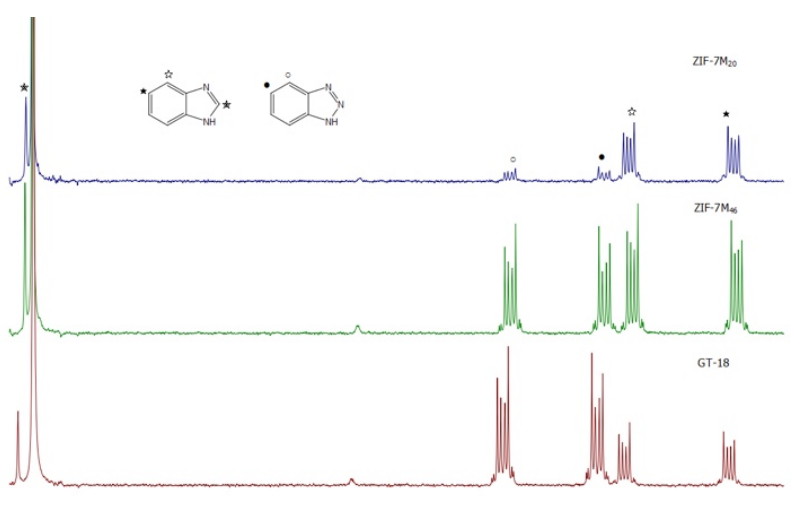

Figure S3. ${ }^{1} \mathrm{H}$ NMR spectra of MOF samples in DCl/D2O (35 wt\%). 


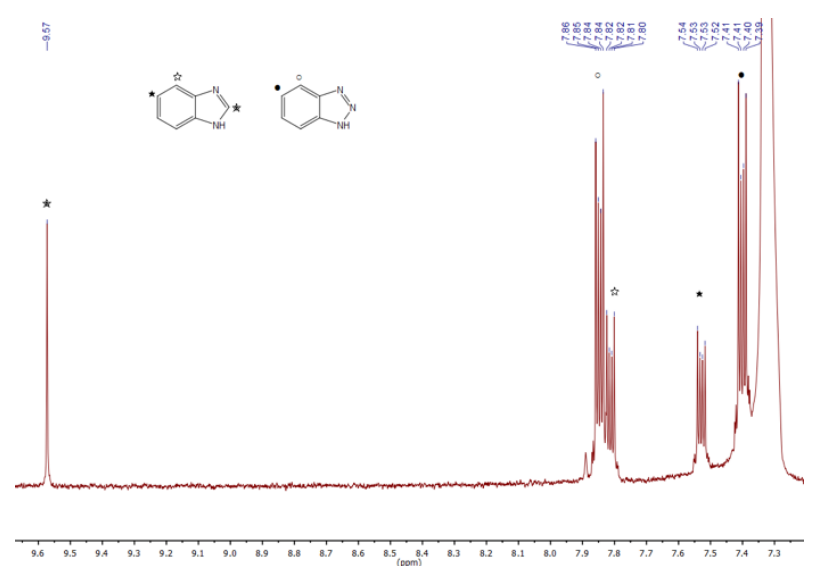

Figure S4. ${ }^{1} \mathrm{H}$ NMR spectrum of GT-18 after digestion in DMSO/DCl/D2O mixture in the range of 7.2-9.7 ppm. The star and circle symbols belong to the BTA and BIM signals, respectively.

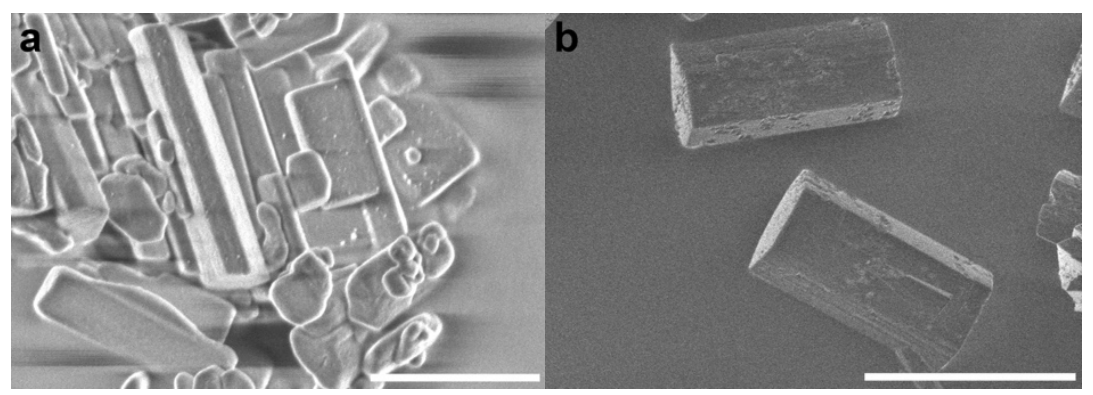

Figure S5. SEM images of small (a) and large (b) GT-18 crystals. GT-18 adopts a rod-like morphology. Scale bars are: (a) $1 \mu \mathrm{m}$ and (b) $50 \mu \mathrm{m}$.

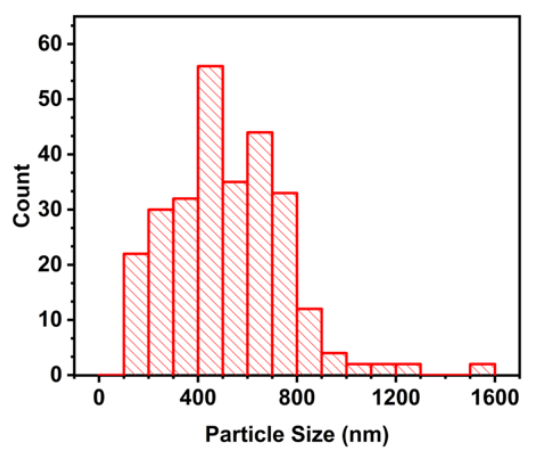

Figure S6. Particle size distribution of the small GT-18 crystals. The sample size for the analysis was 276. 

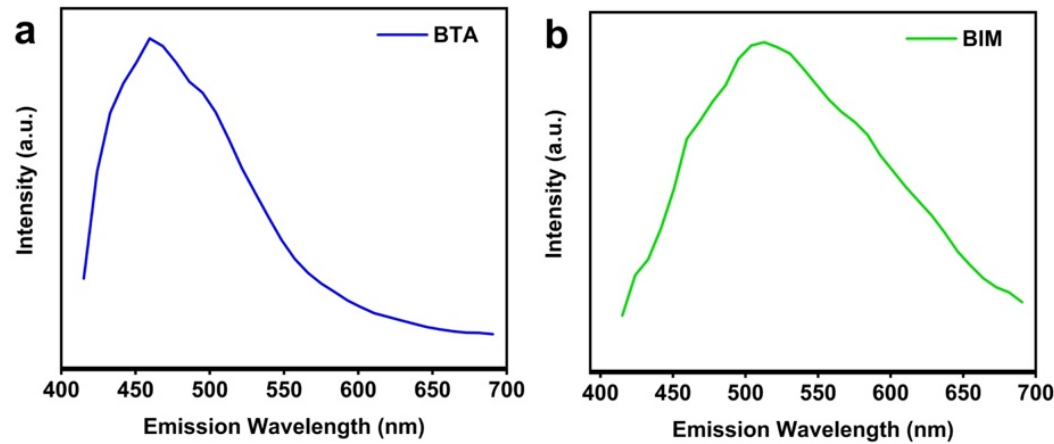

Figure S7. Emission spectra of BTA (a) and BIM (b) excited at $405 \mathrm{~nm}$. The fluorescence emission maxima are at $460 \mathrm{~nm}$ and $510 \mathrm{~nm}$, respectively.

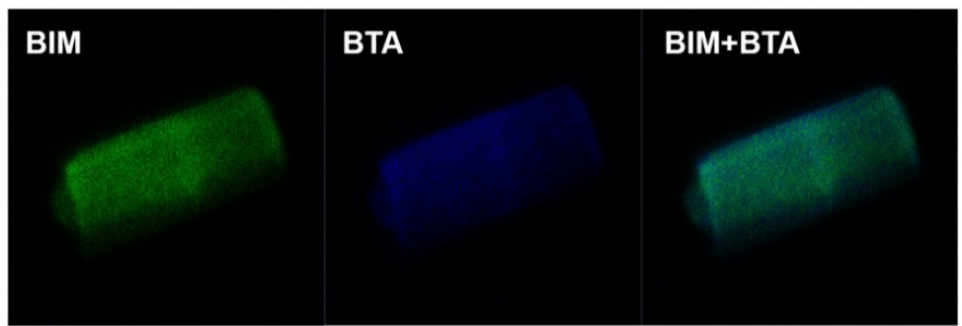

Figure S8. Fluorescence CLSM images of a GT-18 crystal with blue (BTA) and green (BIM) emissions shown separately (left) and combined (right). The fluorescence of both BTA and BIM linkers are equally distributed throughout the surface of the MOF. The images only provide qualitative results as the fluorescence intensities have not been normalized.

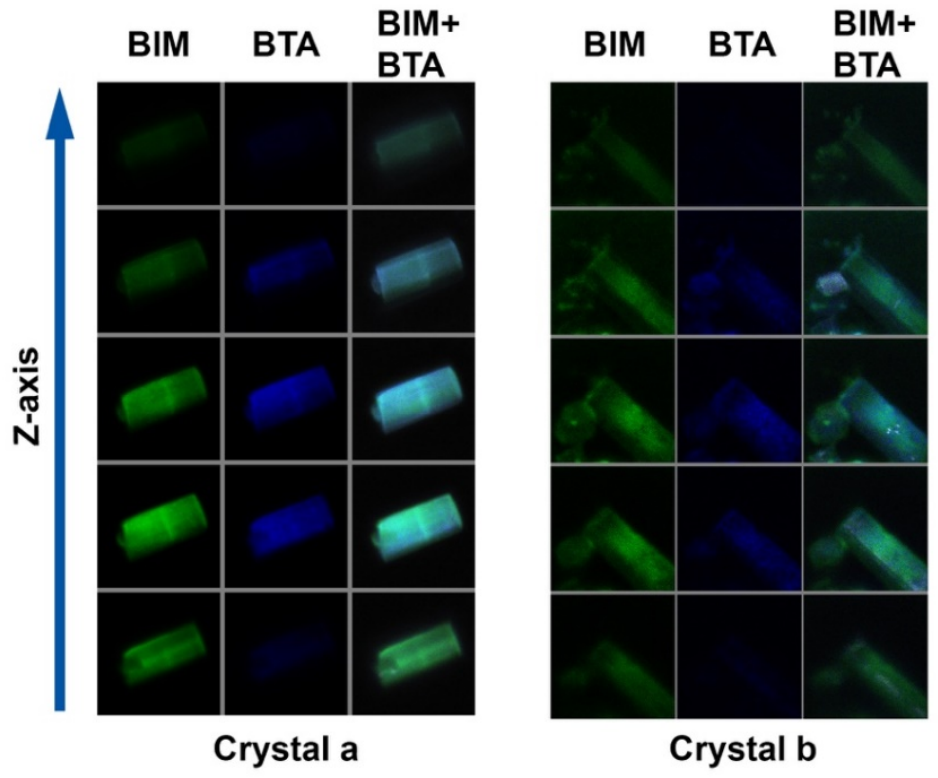

Figure S9. A series of Z-stack CLSM images of GT-18 crystals ( $a$ and b) showing that both BTA (blue emission) and BIM (green emission) are uniformly distributed throughout the entire crosssection of the MOF. Note that BTA is present in every cross-section images (may be difficult to see in some cases due to low contrast issues). The images only provide qualitative results as the fluorescence intensities have not been normalized. 

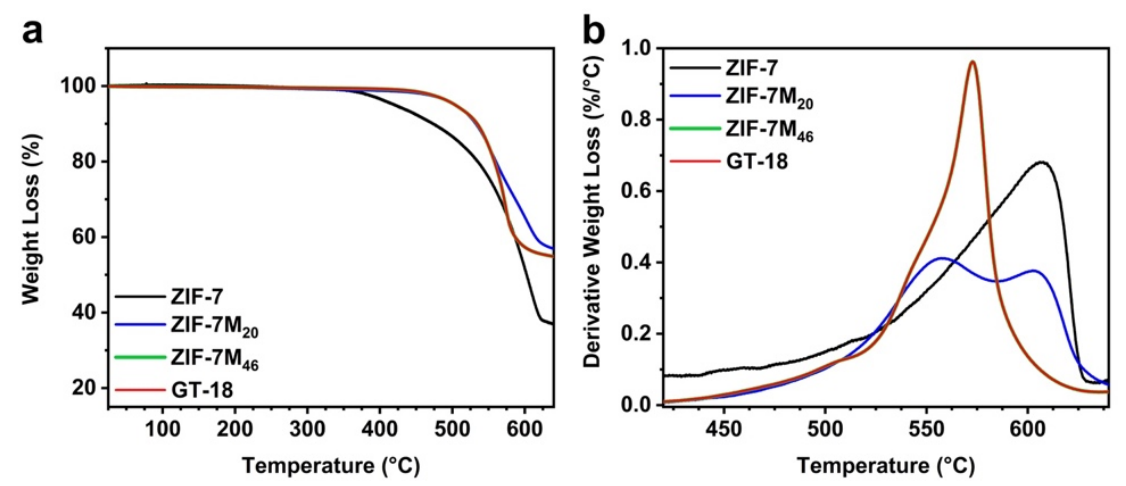

Figure S10. TGA (a) and DTG (b) curves of activated ZIF-7, ZIF-7M $x$ and GT-18. No residual solvent peak can be observed, indicating successful activation. The evacuated frameworks are thermally stable up to $500{ }^{\circ} \mathrm{C}$, where decomposition starts. Sharp decomposition peaks occur at about $570{ }^{\circ} \mathrm{C}$ for ZIF-7M 46 and GT-18, and about $610^{\circ} \mathrm{C}$ for ZIF-7. However, two decomposition peaks can be observed with ZIF-7M 20 at about 560 and $605^{\circ} \mathrm{C}$, which may be attributed to GT-18 and ZIF-7, respectively. This suggests a physical mixture of MOFs is present in the ZIF-7M 20 sample.

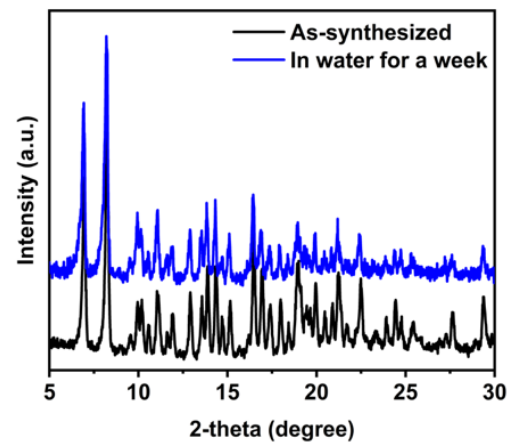

Figure S11. PXRD patterns of GT-18, as prepared and after soaking in water for a week. The sharp peaks were retained with no obvious peak shifts observed after water exposure, suggesting water stability.

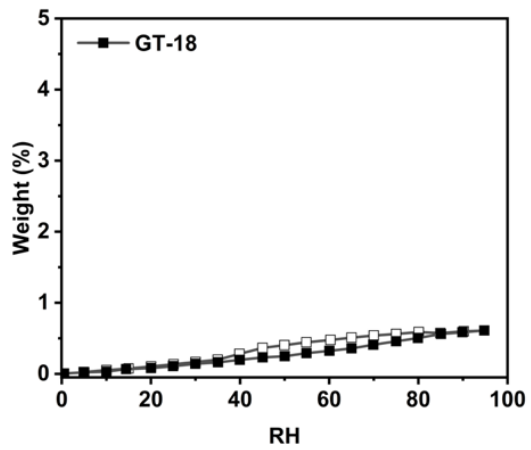

Figure S12. Water vapor adsorption (closed symbol) and desorption (open symbol) isotherms at $298 \mathrm{~K}$. Owing to the hydrophobic nature of GT-18, water can hardly enter the pores despite the small kinetic diameter, resulting in an extremely low water vapor uptake $(\sim 2.7 \AA)$. 


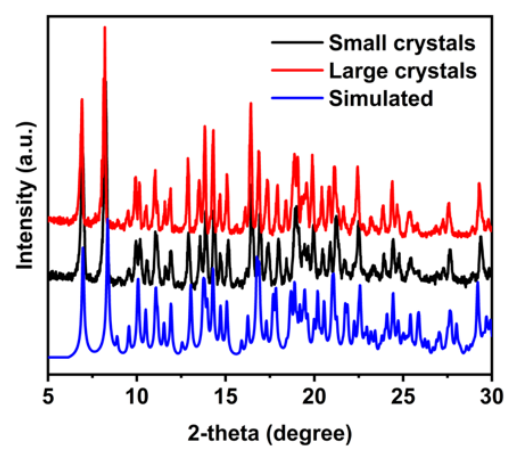

Figure S13. PXRD patterns of small (black) and large (red) GT-18 crystals are consistent with each other. They also correspond well with the simulated pattern calculated using Mercury software (blue). The absence of additional reflections confirms the phase purity of the GT-18 material.

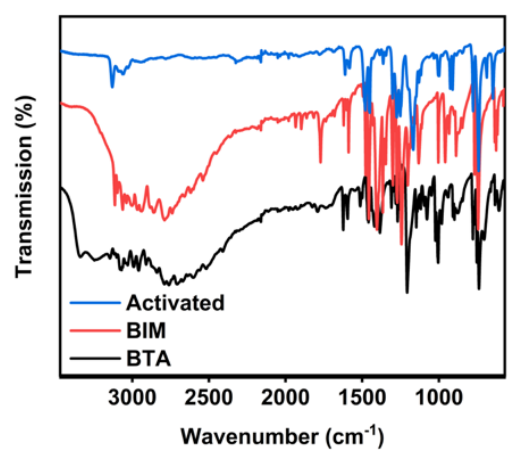

Figure S14. The FT-IR spectra of activated GT-18, BIM and BTA. The GT-18 spectra show both the absence of a broad NH band between $3400-2500 \mathrm{~cm}^{-1} 18-19$ and a $\mathrm{C}=\mathrm{O}$ stretching vibration of residual DMF peak at $1680 \mathrm{~cm}^{-1}$, suggesting the BTA and BIM links in GT-18 have been fully deprotonated and the MOF is fully activated, respectively.

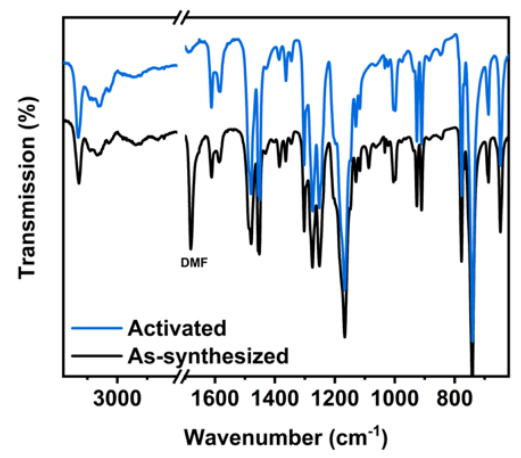

Figure S15. The FT-IR spectra of both activated and as-synthesized GT-18 showing the absence of $\mathrm{C}=\mathrm{O}$ stretching vibration of residual $\mathrm{DMF}$ peak at $1680 \mathrm{~cm}^{-1}$ after activation. 
Table S1. The lattice parameters and unit cell volumes of the crystallographic data of the experimental and DFT predicted structures are comparable with each other, with a slight framework expansion observed in the absence of the DMF molecules.

\begin{tabular}{|l|l|l|l|l|l|l|l|}
\hline $\begin{array}{l}\text { Crystal } \\
\text { Structure }\end{array}$ & $\mathbf{a}(\AA)$ & $\mathbf{b}(\AA)$ & $\mathbf{c}(\AA)$ & $\boldsymbol{\alpha}\left({ }^{\circ}\right)$ & $\boldsymbol{\beta}\left(^{\circ}\right)$ & $\gamma\left(^{\circ}\right)$ & Volume $\left(^{\left.\AA^{3}\right)}\right.$ \\
\hline $\begin{array}{l}\text { Single crystal } \\
\text { (DMF) }\end{array}$ & 9.965 & 15.839 & 21.19 & 90.00 & 94.03 & 90.00 & 3336.12 \\
\hline $\begin{array}{l}\text { DFT relaxed } \\
\text { (DMF) }\end{array}$ & 9.967 & 15.931 & 21.259 & 90.00 & 94.94 & 90.00 & 3362.97 \\
\hline $\begin{array}{l}\text { DFT relaxed (no } \\
\text { DMF) }\end{array}$ & 10.015 & 15.935 & 21.306 & 90.00 & 90.00 & 90.00 & 3391.27 \\
\hline
\end{tabular}
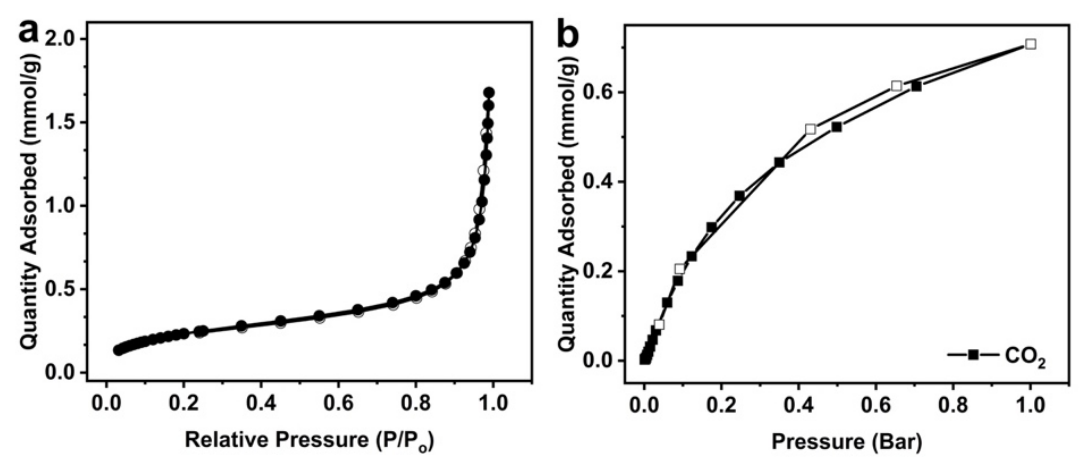

Figure S16. Single component adsorption isotherms of nitrogen at $77 \mathrm{~K}$ (a) and carbon dioxide at $298 \mathrm{~K}$ (b) to confirm the porosity of GT- 18.
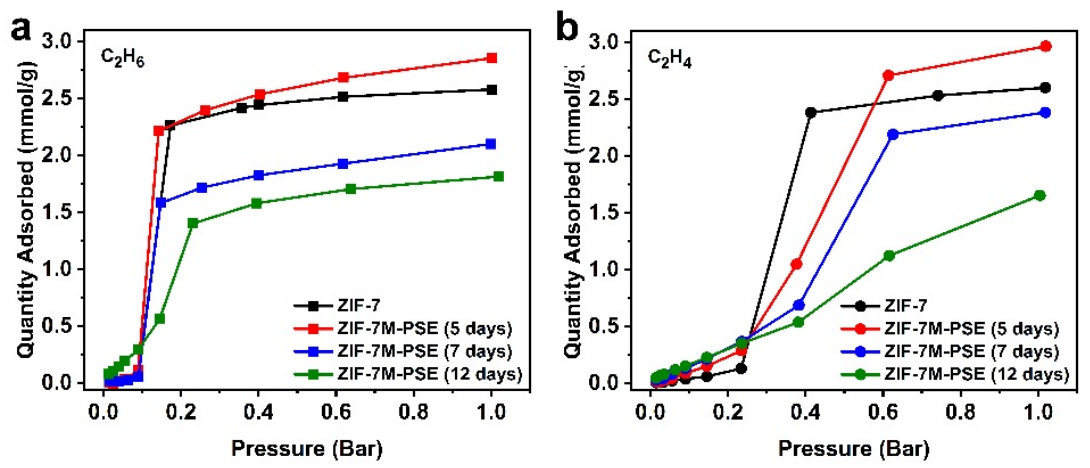

Figure S17. Single component adsorption isotherms of ethane (a) and ethylene (b) in ZIF-7 and ZIF-7M-PSE at $298 \mathrm{~K}$. ZIF-7-M-PSE prepared via 5, 7 and 12-day ligand exchange contain approximately $17 \%, 26 \%, 52 \%$ BTA, respectively. BTA contents were determined by ${ }^{1} \mathrm{H}$ NMR. Gate opening characteristics can be observed in all ZIF-7M-PSE samples. 


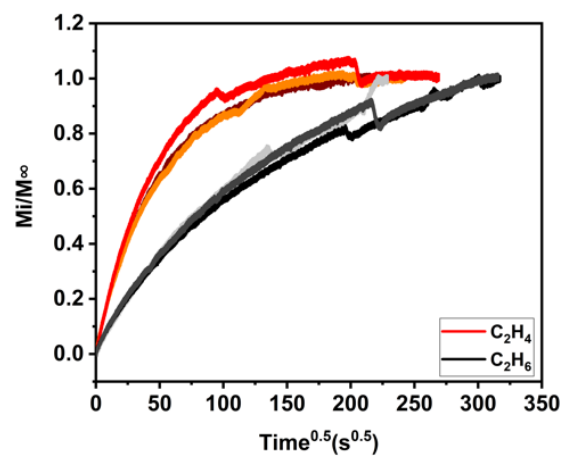

Figure S18. The kinetic uptake plots of ethylene (red) and ethane (black) in GT-18 at $298 \mathrm{~K}$ at 0.05 bar. The uptakes were measured in triplicates to ensure reproducibility (same sample batch).

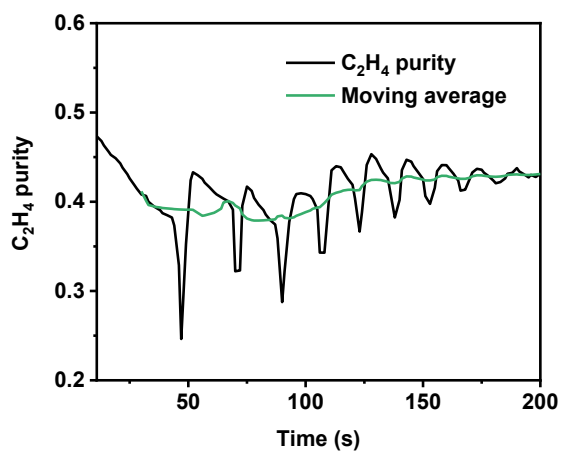

Figure S19. Batch adsorption and average ethylene purity profiles of GT-18 at $20{ }^{\circ} \mathrm{C}$ in equimolar ethylene/ethane mixture. The experiment was conducted for $2.5 \mathrm{~h}$, which was operating within the kinetic regime (ethane not in equilibrium). More ethylene was adsorbed by GT-18 since there is no sorption selectivity, but high ethylene/ethane diffusion selectivity. The average purity profile of ethylene is $\sim 0.5$ The fluctuations in the purity profile are due to pressure issues with the mass spectrometer, and the moving average shown in green is indicative of the system's performance.

Table S2. Calculated diffusivity values for ethane and ethylene in GT-18 at $298 \mathrm{~K}$ by modelling the uptake curves shown in Figure S18. The kinetic diameters of ethylene and ethane are $4.2 \AA$ and $4.4 \AA$. The scale of kinetic diameters is used to characterize molecular dimension as it has been shown to be suitable for explaining the molecular sieving behavior of zeolites for simple diffusing molecules as well as linear and branched paraffins. ${ }^{20}$

\begin{tabular}{|l|l|c|c|}
\hline Gases & Diffusivities $\left(\mathrm{cm}^{2} / \mathbf{s}\right)$ & Average.Diffusivity $\left(\mathrm{cm}^{2} / \mathbf{s}\right)$ & Standard Deviation $\left(\mathrm{cm}^{2} / \mathbf{s}\right)$ \\
\hline $\mathrm{C}_{2} \mathrm{H}_{4}$ & $2.50 \times 10^{-14}$ & $2.00 \times 10^{-14}$ & $5.00 \times 10^{-15}$ \\
\hline & $2.00 \times 10^{-14}$ & & \\
\hline & $1.50 \times 10^{-14}$ & $2.93 \times 10^{-15}$ & $5.13 \times 10^{-16}$ \\
\hline $\mathrm{C}_{2} \mathrm{H}_{6}$ & $2.50 \times 10^{-15}$ & & \\
\hline & $3.50 \times 10^{-15}$ & & \\
\hline & $2.80 \times 10^{-15}$ & & \\
\hline
\end{tabular}


Table S3. Ethylene/ethane diffusion selectivity in porous materials determined experimentally. Note that ethylene/ethane and propylene/propane separations are important in the petrochemical industry. The size differences for both olefin-paraffin pairs are small. However, compared to ethylene and ethane, membrane separation is more practical for propylene and propane due to the slightly larger difference in diffusion coefficients between propylene and propane. Hence improving the ethylene/ethane selectivity is important for membrane applications. ${ }^{21}$

\begin{tabular}{|l|l|l|l|}
\hline Material & Class & $\begin{array}{l}\mathbf{C}_{2} \mathbf{H}_{4} / \mathbf{C}_{2} \mathbf{H}_{6} \\
\text { selectivity }\end{array}$ & Method \\
\hline ITQ-55 & Zeolite & 90 & Pressure decay $^{22}$ \\
\hline Al-ITQ-55 & Zeolite & 300 & Pressure decay $^{22}$ \\
\hline DDR & Zeolite & 10 & IR microscopy $^{23}$ \\
\hline GT-18 (this work) & MOF & 6.8 & Pressure decay $^{24}$ \\
\hline $33 \% \mathrm{Co}_{2}$ (dobdc)/6FDA-DAM & MOF & 3.4 & Time-lag method $^{24}$ \\
\hline $25 \% \mathrm{Ni}_{2}($ dobdc)/6FDA-DAM & MOF & 3.6 & Time-lag method $^{24}$ \\
\hline ZIF-8 & MOF & 5.5 & IR microscopy $^{25}$ \\
\hline ZIF-7 & MOF & 0.4 & Zero-length column technique $^{26}$ \\
\hline Mg-MOF-74 & MOF & 0.5 & Pressure decay $^{24}$ \\
\hline
\end{tabular}




\section{Section 3: Single Crystal X-Ray Crystallography}

Single GT-18 crystals were selected and analyzed using an XtaLAB Synergy-S diffractometer with a Hypix-6000HE Hybrid Photon Counting (HPC) detector. The crystal was held at a constant $\mathrm{T}=104(1) \mathrm{K}$ during data collection. The structure was solved with the ShelXT ${ }^{27}$ structure solution program using the Intrinsic Phasing solution method and by using Olex $2{ }^{28}$ as the graphical interface. The crystal structure was refined with version 2018/3 of SHELXL ${ }^{29}$ using Least Squares minimization. All non-hydrogen atoms were refined anisotropically. Structure simplification and net classification were performed using the TOPOS program suite ${ }^{30}$.
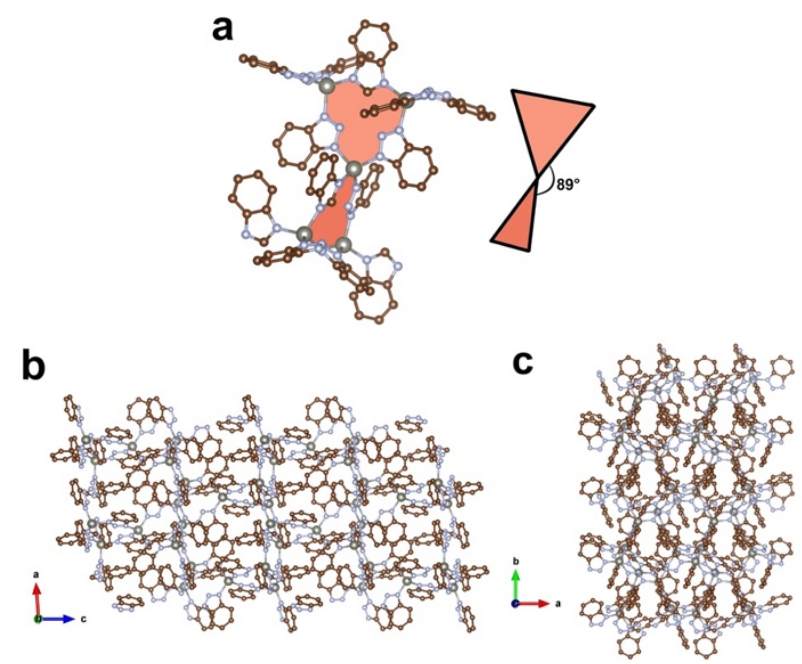

Figure S20. (a) The coordination environment of $\mathrm{Zn} 2+$, featuring one of the zinc-azolate units as a "twisted strut," with a twist angle of almost $90^{\circ}$ between the two central planes that connect pairs of zinc-azolate units. The structure viewed along the (b) $b$-axis and (c) $c$-axis. Hydrogen atoms and DMF molecules located in the structure are omitted for clarity.

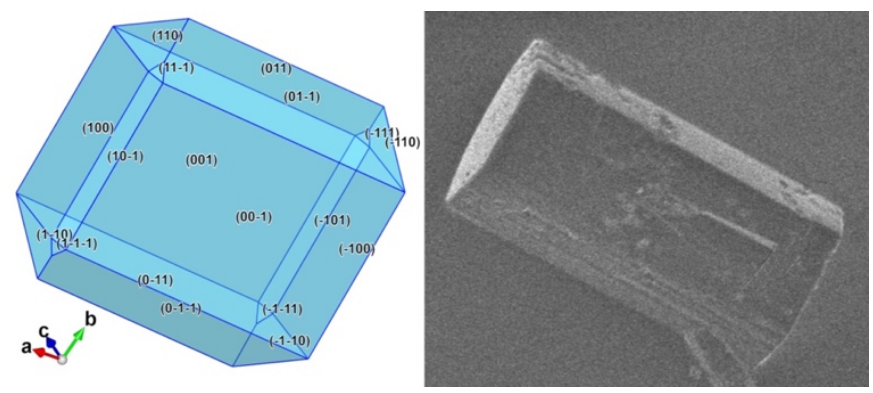

Figure S21. Simulated crystal morphology of and an SEM image of GT-18 crystal with crystallographic faces and axes labeled, with faceting observed primarily on the [001], [010] and [100] faces. The $a$-axis (main diffusion path) is along the longest dimension of the crystals. Bravais-Friedel-Donnay-Harker (BFDH) method is applied for crystal morphology simulation using the Mercury software. The lattice parameters and atomic coordinates used in the model were obtained from experimentally determined single crystal data. 

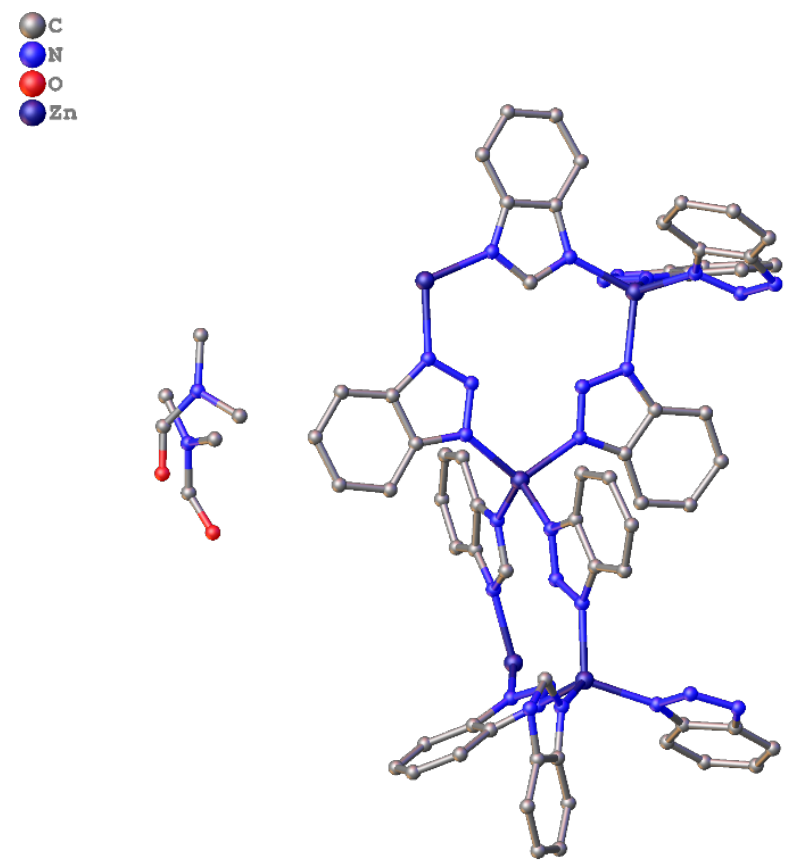

Figure S22. Asymmetric unit of the GT-18 MOF. The MOF was not activated and DMF molecules are present in the framework. 
Table S5. Crystal data and structure refinement for GT-18.

\begin{tabular}{|c|c|}
\hline Empirical formula & $\mathrm{C}_{66} \mathrm{H}_{50} \mathrm{~N}_{28} \mathrm{OZn}_{5}$ \\
\hline Formula weight & 1578.19 \\
\hline Temperature/K & 104(1) \\
\hline Crystal system & monoclinic \\
\hline Space group & $\mathrm{P} 2_{1}$ \\
\hline $\mathrm{a} / \AA$ & $9.96450(10)$ \\
\hline $\mathrm{b} / \AA \AA$ & $15.83910(10)$ \\
\hline $\mathrm{c} / \AA$ & $21.1899(2)$ \\
\hline$\alpha /{ }^{\circ}$ & 90 \\
\hline$\beta /{ }^{\circ}$ & $94.0260(10)$ \\
\hline$\gamma /{ }^{\circ}$ & 90 \\
\hline Volume $/ \AA^{3}$ & $3336.12(5)$ \\
\hline Z & 2 \\
\hline$\rho_{\text {calc }} \mathrm{g} / \mathrm{cm}^{3}$ & 1.571 \\
\hline$\mu / \mathrm{mm}^{-1}$ & 2.552 \\
\hline $\mathrm{F}(000)$ & 1600.0 \\
\hline Crystal size $/ \mathrm{mm}^{3}$ & $0.085 \times 0.057 \times 0.031$ \\
\hline Radiation & $\mathrm{CuK} \alpha(\lambda=1.54184)$ \\
\hline \multicolumn{2}{|c|}{$2 \Theta$ range for data collection $/{ }^{\circ} 6.974$ to 152.75} \\
\hline Index ranges & $-11 \leq \mathrm{h} \leq 12,-19 \leq \mathrm{k} \leq 13,-26 \leq 1 \leq 26$ \\
\hline Reflections collected & 24553 \\
\hline Independent reflections & $10214\left[R_{\text {int }}=0.0468, R_{\text {sigma }}=0.0566\right]$ \\
\hline Data/restraints/parameters & $10214 / 44 / 920$ \\
\hline Goodness-of-fit on $\mathrm{F}^{2}$ & 1.039 \\
\hline Final $R$ indexes $[I>=2 \sigma(I)]$ & $\mathrm{R}_{1}=0.0407, \mathrm{wR}_{2}=0.0956$ \\
\hline Final $\mathrm{R}$ indexes [all data] & $\mathrm{R}_{1}=0.0448, \mathrm{wR}_{2}=0.0981$ \\
\hline \multicolumn{2}{|c|}{ Largest diff. peak/hole / e $\AA^{-3} 0.77 /-0.61$} \\
\hline Flack parameter & $0.02(2)$ \\
\hline
\end{tabular}

Table S6. Fractional Atomic Coordinates $\left(\times 10^{4}\right)$ and Equivalent Isotropic Displacement Parameters $\left(\AA^{2} \times 10^{3}\right)$ for $126-6$. $U_{\text {eq }}$ is defined as $1 / 3$ of of the trace of the orthogonalised $U_{I J}$ tensor.

$\begin{array}{lllll}\text { Atom } & \underline{\boldsymbol{x}} & \boldsymbol{y} & \underline{\mathbf{z}} & \text { U(eq) } \\ \text { Zn1 } & 8800.2(7) & -433.8(5) & 0.8(3) & 17.37(17) \\ \text { Zn2 } & 8036.3(9) & 679.5(5) & 2570.6(4) & 24.68(19) \\ \text { Zn3 } & 11303.5(8) & 250.6(5) & 4930.6(4) & 20.25(18) \\ \text { Zn4 } & 6248.7(8) & -1364.9(5) & 4603.7(4) & 21.42(18) \\ \text { Zn5 } & 6338.6(7) & 2851.5(5) & 443.4(3) & 16.81(16) \\ \text { N1 } & 7622(5) & -1411(3) & -283(2) & 21.4(10) \\ \text { N2 } & 10668(5) & -491(3) & -294(2) & 22.4(10)\end{array}$




\begin{tabular}{|c|c|c|c|c|}
\hline N3 & $11358(5)$ & $-1200(3)$ & $-170(2)$ & $21.7(10)$ \\
\hline N4 & $12639(5)$ & $-1090(3)$ & $-307(2)$ & $19.7(10)$ \\
\hline N5 & $9016(5)$ & $-530(3)$ & $952(2)$ & $21.9(10)$ \\
\hline N6 & $8556(6)$ & $93(3)$ & $1287(2)$ & $26.5(11)$ \\
\hline N7 & $8562(6)$ & $-119(3)$ & $1892(2)$ & $28.0(12)$ \\
\hline N8 & $9685(6)$ & $782(3)$ & $3154(2)$ & $28.4(12)$ \\
\hline N9 & $9780(5)$ & $453(4)$ & $3735(2)$ & $27.5(12)$ \\
\hline N10 & $11006(5)$ & $537(3)$ & $4016(2)$ & $22.3(11)$ \\
\hline N11 & $9503(5)$ & $-4(3)$ & $5258(2)$ & $22.5(11)$ \\
\hline N12 & $8796(5)$ & $-612(3)$ & $4948(2)$ & $23.1(11)$ \\
\hline N13 & $7547(5)$ & $-633(3)$ & $5125(2)$ & $21.6(10)$ \\
\hline N14 & $5922(5)$ & $-712(3)$ & $3789(2)$ & $21.6(10)$ \\
\hline N15 & $6637(6)$ & $60(3)$ & $3018(2)$ & $29.9(12)$ \\
\hline N16 & $12488(5)$ & $-772(3)$ & $5043(2)$ & $19.3(10)$ \\
\hline N17 & $13753(5)$ & $-650(3)$ & $4895(2)$ & $23.0(11)$ \\
\hline N18 & $14430(5)$ & $-1382(3)$ & $4938(2)$ & $21.5(10)$ \\
\hline N19 & $12179(5)$ & $1216(3)$ & $5394(2)$ & $21.6(10)$ \\
\hline $\mathrm{N} 20$ & $13031(5)$ & $2500(3)$ & $5591(2)$ & $21.1(10)$ \\
\hline $\mathrm{N} 21$ & $7387(5)$ & 1761(3) & $2182(2)$ & $22.5(11)$ \\
\hline N22 & $6649(5)$ & $2550(3)$ & 1371(2) & $20.7(10)$ \\
\hline $\mathrm{N} 23$ & $7048(5)$ & $1932(3)$ & $-86(2)$ & $20.0(10)$ \\
\hline N24 & $7878(5)$ & $655(3)$ & $-241(2)$ & $19.4(10)$ \\
\hline $\mathrm{N} 25$ & $6310(5)$ & $-1315(4)$ & $-213(3)$ & $23.5(10)$ \\
\hline N26 & $5666(5)$ & $-2031(3)$ & $-357(2)$ & $19.7(10)$ \\
\hline $\mathrm{C} 1$ & $6429(7)$ & $-3462(4)$ & $-718(3)$ & $25.1(13)$ \\
\hline $\mathrm{C} 2$ & $7582(7)$ & $-3879(4)$ & $-852(3)$ & $28.5(14)$ \\
\hline $\mathrm{C} 3$ & $8864(7)$ & $-3480(4)$ & $-811(3)$ & $28.4(14)$ \\
\hline $\mathrm{C} 4$ & $8997(6)$ & $-2655(4)$ & $-628(3)$ & $23.3(12)$ \\
\hline C5 & $7842(5)$ & $-2220(4)$ & $-480(3)$ & $19.0(11)$ \\
\hline C6 & $12785(6)$ & $-287(4)$ & $-529(3)$ & $18.7(12)$ \\
\hline $\mathrm{C} 7$ & $13906(6)$ & $155(4)$ & $-729(3)$ & $22.3(12)$ \\
\hline $\mathrm{C} 8$ & $13674(6)$ & $960(4)$ & $-935(3)$ & $26.7(13)$ \\
\hline C9 & $12385(6)$ & $1350(4)$ & $-940(3)$ & $27.3(14)$ \\
\hline $\mathrm{C} 10$ & $11306(6)$ & $934(4)$ & $-728(3)$ & $23.8(13)$ \\
\hline $\mathrm{C} 11$ & $11510(5)$ & $94(4)$ & $-524(3)$ & $17.2(11)$ \\
\hline $\mathrm{C} 12$ & $6910(6)$ & $-241(3)$ & $3600(2)$ & $16.8(11)$ \\
\hline $\mathrm{C} 13$ & $5381(8)$ & $-243(4)$ & $2817(3)$ & $35.5(17)$ \\
\hline C14 & 4591(11) & $-143(6)$ & $2252(5)$ & $67(3)$ \\
\hline C15 & $3385(12)$ & $-543(7)$ & $2193(5)$ & $84(4)$ \\
\hline $\mathrm{C} 16$ & 2913(10) & $-1014(6)$ & $2692(6)$ & $75(4)$ \\
\hline $\mathrm{C} 17$ & $3671(8)$ & $-1117(5)$ & $3259(4)$ & $45(2)$ \\
\hline C18 & $4930(7)$ & $-725(4)$ & $3310(3)$ & $30.4(15)$ \\
\hline C19 & $7408(6)$ & $-9(4)$ & $5569(3)$ & $22.6(12)$ \\
\hline
\end{tabular}




\begin{tabular}{|c|c|c|c|c|}
\hline $\mathrm{C} 20$ & $6283(6)$ & $265(4)$ & $5881(3)$ & $24.5(13)$ \\
\hline $\mathrm{C} 21$ & $6487(7)$ & $936(5)$ & $6276(3)$ & $31.7(15)$ \\
\hline $\mathrm{C} 22$ & $7733(7)$ & $1345(4)$ & $6364(3)$ & $28.4(14)$ \\
\hline $\mathrm{C} 23$ & $8838(7)$ & $1086(4)$ & $6064(3)$ & $26.6(13)$ \\
\hline $\mathrm{C} 24$ & $8654(6)$ & $400(4)$ & $5655(3)$ & $19.7(12)$ \\
\hline $\mathrm{C} 25$ & $13573(6)$ & $-1994(4)$ & $5114(3)$ & $18.9(12)$ \\
\hline $\mathrm{C} 26$ & $13759(6)$ & $-2857(4)$ & $5237(3)$ & $23.4(13)$ \\
\hline $\mathrm{C} 27$ & $12649(7)$ & $-3299(4)$ & $5414(3)$ & $27.7(14)$ \\
\hline $\mathrm{C} 28$ & $11412(7)$ & $-2908(4)$ & $5467(3)$ & $30.4(15)$ \\
\hline C29 & $11212(7)$ & $-2062(4)$ & $5350(3)$ & $28.8(14)$ \\
\hline $\mathrm{C} 30$ & $12317(6)$ & $-1605(4)$ & $5173(3)$ & $21.8(12)$ \\
\hline C31 & $12503(6)$ & $1965(4)$ & $5157(3)$ & $17.6(11)$ \\
\hline $\mathrm{C} 32$ & $13053(6)$ & $2066(4)$ & $6164(3)$ & $20.0(12)$ \\
\hline C33 & $13458(6)$ & $2306(4)$ & $6782(3)$ & $24.5(13)$ \\
\hline C34 & $13321(7)$ & $1722(4)$ & $7256(3)$ & $28.5(14)$ \\
\hline $\mathrm{C} 35$ & $12800(7)$ & $898(4)$ & $7115(3)$ & $34.0(16)$ \\
\hline $\mathrm{C} 36$ & $12397(6)$ & $671(4)$ & $6515(3)$ & $28.3(13)$ \\
\hline C37 & $12531(6)$ & $1264(4)$ & $6035(3)$ & $20.8(12)$ \\
\hline C38 & $11781(7)$ & $939(4)$ & $3593(3)$ & $28.2(14)$ \\
\hline C39 & $10938(8)$ & $1102(4)$ & $3050(3)$ & $32.3(15)$ \\
\hline $\mathrm{C} 40$ & $11397(12)$ & $1508(6)$ & $2521(4)$ & $57(3)$ \\
\hline C41 & $12729(14)$ & $1736(7)$ & $2572(5)$ & $80(4)$ \\
\hline $\mathrm{C} 42$ & $13608(12)$ & $1549(8)$ & $3114(5)$ & $81(4)$ \\
\hline $\mathrm{C} 43$ & $13155(8)$ & 1161(6) & $3640(4)$ & $50(2)$ \\
\hline N44 & $7277(6)$ & $1836(4)$ & $1553(3)$ & $27.4(12)$ \\
\hline $\mathrm{C} 45$ & $7597(6)$ & $1244(3)$ & $178(3)$ & $14.9(10)$ \\
\hline $\mathrm{C} 46$ & $7455(6)$ & $989(4)$ & $-828(3)$ & $21.4(12)$ \\
\hline $\mathrm{C} 47$ & $6968(6)$ & $1795(4)$ & $-724(3)$ & $19.4(12)$ \\
\hline $\mathrm{C} 48$ & $6482(6)$ & $2305(4)$ & $-1234(3)$ & $25.1(13)$ \\
\hline C49 & $6482(7)$ & 1961(5) & $-1824(3)$ & $31.5(16)$ \\
\hline C50 & $6948(7)$ & $1143(5)$ & $-1922(3)$ & $34.4(16)$ \\
\hline C51 & $7456(6)$ & $640(5)$ & $-1437(3)$ & $28.8(13)$ \\
\hline C52 & $6308(6)$ & $2960(4)$ & 1908(3) & $20.8(12)$ \\
\hline C53 & $5644(7)$ & $3714(4)$ & 2001(3) & $32.1(15)$ \\
\hline C54 & $5486(8)$ & $3964(5)$ & $2613(3)$ & $39.3(17)$ \\
\hline C55 & $5981(7)$ & $3474(4)$ & $3123(3)$ & $33.1(15)$ \\
\hline C56 & $6651(7)$ & $2722(4)$ & $3048(3)$ & $27.1(13)$ \\
\hline C57 & $6791(6)$ & $2464(4)$ & $2422(3)$ & $20.9(12)$ \\
\hline C58 & $9047(6)$ & $-932(4)$ & $1954(3)$ & $22.3(12)$ \\
\hline C59 & $9228(7)$ & $-1459(4)$ & $2485(3)$ & $26.3(13)$ \\
\hline C60 & $9745(7)$ & $-2253(4)$ & $2375(3)$ & $30.3(14)$ \\
\hline C61 & $10114(6)$ & $-2493(4)$ & $1777(3)$ & $29.5(14)$ \\
\hline C62 & $9934(6)$ & $-1984(4)$ & $1256(3)$ & $26.3(13)$ \\
\hline
\end{tabular}




$\begin{array}{lllll}\text { C63 } & 9357(5) & -1184(3) & 1357(3) & 18.3(11) \\ \text { C64 } & 6576(6) & -2617(4) & -526(3) & 18.6(11) \\ \text { O5S_1 } & 1347(13) & 3062(9) & 3791(6) & 108(4) \\ \text { N1S_1 } & 739(12) & 3723(9) & 2783(6) & 92(3) \\ \text { C2S_1 } & -197(18) & 3879(17) & 2241(8) & 134(8) \\ \text { C3S_1 } & 2088(14) & 4028(12) & 2743(9) & 96(5) \\ \text { C4S_1 } & 580(18) & 3332(12) & 3355(7) & 99(4) \\ \text { O5S_2 } & 20(40) & 3680(30) & 3180(13) & 108(4) \\ \text { N1S_2 } & 860(30) & 3870(20) & 2146(12) & 92(3) \\ \text { C2S_2 } & 650(50) & 3970(40) & 1467(12) & 134(8) \\ \text { C3S_2 } & 2200(30) & 3650(40) & 2391(19) & 96(5) \\ \text { C4S_2 } & -30(30) & 3880(30) & 2617(13) & 99(4)\end{array}$

Table S7. Anisotropic Displacement Parameters $\left(\AA^{2} \times 10^{3}\right)$ for $126-6$. The Anisotropic displacement factor exponent takes the form: $-2 \pi^{2}\left[h^{2} a *{ }^{2} U_{11}+2 h k a * b * U_{12}+\ldots\right]$.

$\begin{array}{lllllll}\text { Atom } & \mathbf{U}_{\mathbf{1 1}} & \mathbf{U}_{\mathbf{2 2}} & \mathbf{U}_{\mathbf{3 3}} & \mathbf{U}_{\mathbf{2 3}} & \mathbf{U}_{\mathbf{1 3}} & \mathbf{U}_{\mathbf{1 2}} \\ \text { Zn1 } & 13.9(4) & 14.1(4) & 24.7(4) & -1.4(3) & 4.7(3) & -0.6(3) \\ \text { Zn2 } & 33.6(5) & 19.8(4) & 20.7(4) & 3.2(3) & 3.0(3) & 8.6(4) \\ \text { Zn3 } & 16.9(4) & 17.9(4) & 25.8(4) & -4.5(3) & 0.5(3) & 0.5(3) \\ \text { Zn4 } & 18.8(4) & 16.3(4) & 29.7(4) & -0.4(3) & 5.9(3) & 0.2(3) \\ \text { Zn5 } & 13.9(4) & 14.2(4) & 22.6(4) & 2.0(3) & 2.9(3) & 2.3(3) \\ \text { N1 } & 13(2) & 20(3) & 31(3) & -5(2) & 5.4(18) & -1(2) \\ \text { N2 } & 18(2) & 18(3) & 31(3) & 0(2) & 6.1(19) & 1(2) \\ \text { N3 } & 14(2) & 17(3) & 35(3) & 1(2) & 9.2(19) & -0.7(19) \\ \text { N4 } & 14(2) & 17(2) & 30(2) & 0(2) & 8.0(19) & -1.3(19) \\ \text { N5 } & 27(3) & 13(2) & 27(2) & -1(2) & 4.8(19) & 1(2) \\ \text { N6 } & 38(3) & 18(3) & 25(2) & 1(2) & 8(2) & 5(2) \\ \text { N7 } & 44(3) & 16(3) & 24(3) & 4(2) & 7(2) & 12(2) \\ \text { N8 } & 35(3) & 24(3) & 26(3) & 2(2) & 5(2) & 10(2) \\ \text { N9 } & 22(3) & 34(3) & 27(2) & 0(2) & -1.3(19) & 6(2) \\ \text { N10 } & 17(2) & 24(3) & 26(2) & -6(2) & 3.5(19) & 1(2) \\ \text { N11 } & 17(2) & 25(3) & 25(2) & -6(2) & 0.9(19) & 2(2) \\ \text { N12 } & 17(2) & 26(3) & 26(3) & -4(2) & 3.4(19) & 2(2) \\ \text { N13 } & 16(2) & 24(3) & 25(2) & 1(2) & 5.9(18) & 1(2) \\ \text { N14 } & 21(3) & 17(2) & 26(2) & -2(2) & 0.6(19) & 1(2) \\ \text { N15 } & 39(3) & 23(3) & 27(3) & 5(2) & -1(2) & 9(2) \\ \text { N16 } & 16(2) & 17(2) & 26(2) & -0.4(19) & 3.7(19) & -0.3(19) \\ \text { N17 } & 19(2) & 15(3) & 35(3) & -1(2) & 7(2) & 5(2) \\ \text { N18 } & 15(2) & 15(2) & 35(3) & 2(2) & 5.0(19) & 2(2) \\ \text { N19 } & 21(2) & 18(3) & 26(2) & 2(2) & 2.3(19) & 1(2) \\ \text { N20 } & 20(2) & 22(3) & 23(2) & 0(2) & 6.9(19) & 1(2)\end{array}$




\begin{tabular}{|c|c|c|c|c|c|c|}
\hline N21 & $26(3)$ & $21(3)$ & $21(2)$ & $5.7(19)$ & $0.9(19)$ & $8(2)$ \\
\hline N22 & $22(2)$ & $13(2)$ & $27(2)$ & $4.0(19)$ & $3.6(19)$ & $6.0(19)$ \\
\hline $\mathrm{N} 23$ & $15(2)$ & $15(2)$ & $30(3)$ & $5(2)$ & $-0.2(19)$ & $2(2)$ \\
\hline N24 & $17(2)$ & $18(2)$ & $24(2)$ & $0(2)$ & $4.8(18)$ & $0(2)$ \\
\hline $\mathrm{N} 25$ & $15(2)$ & $20(2)$ & $36(3)$ & $-6(2)$ & $1.4(18)$ & $-2(2)$ \\
\hline N26 & $13(2)$ & $17(2)$ & $30(2)$ & $-4(2)$ & $3.1(18)$ & $-3.3(19)$ \\
\hline $\mathrm{C} 1$ & $27(3)$ & $19(3)$ & $29(3)$ & $-4(2)$ & $2(2)$ & $-3(3)$ \\
\hline $\mathrm{C} 2$ & $31(4)$ & $18(3)$ & $35(3)$ & $-9(3)$ & $-4(3)$ & $4(3)$ \\
\hline $\mathrm{C} 3$ & $29(3)$ & $25(3)$ & $31(3)$ & $-8(3)$ & $-1(3)$ & $8(3)$ \\
\hline $\mathrm{C} 4$ & $19(3)$ & $23(3)$ & $29(3)$ & $-1(3)$ & $7(2)$ & $5(2)$ \\
\hline $\mathrm{C} 5$ & $18(3)$ & $17(3)$ & $22(3)$ & $3(2)$ & $1(2)$ & $3(2)$ \\
\hline C6 & $17(3)$ & $14(3)$ & $25(3)$ & $-4(2)$ & $5(2)$ & $-5(2)$ \\
\hline $\mathrm{C} 7$ & $15(3)$ & $21(3)$ & $32(3)$ & $-1(2)$ & $8(2)$ & $-1(2)$ \\
\hline $\mathrm{C} 8$ & $18(3)$ & $29(3)$ & $33(3)$ & $4(3)$ & $6(2)$ & $-5(3)$ \\
\hline C9 & $22(3)$ & $19(3)$ & $41(4)$ & $5(3)$ & $-1(3)$ & $-4(3)$ \\
\hline $\mathrm{C} 10$ & $17(3)$ & $26(3)$ & $29(3)$ & $1(2)$ & $4(2)$ & $3(2)$ \\
\hline C11 & $13(3)$ & $16(3)$ & $23(3)$ & $-1(2)$ & $4(2)$ & $-3(2)$ \\
\hline C12 & $21(3)$ & $16(3)$ & $14(2)$ & $2(2)$ & $2(2)$ & $-2(2)$ \\
\hline C13 & $44(4)$ & $22(4)$ & $38(4)$ & $6(3)$ & $-13(3)$ & $0(3)$ \\
\hline C14 & $90(8)$ & $46(5)$ & $58(5)$ & $28(4)$ & $-37(5)$ & $-23(5)$ \\
\hline C15 & $90(8)$ & $64(7)$ & $87(7)$ & $28(6)$ & $-68(7)$ & $-18(6)$ \\
\hline C16 & $60(6)$ & $45(5)$ & 109(8) & $16(6)$ & $-57(6)$ & $-12(5)$ \\
\hline $\mathrm{C} 17$ & $32(4)$ & $27(4)$ & $74(5)$ & $5(4)$ & $-21(4)$ & $0(3)$ \\
\hline C18 & $28(3)$ & $16(3)$ & $46(4)$ & $0(3)$ & $-11(3)$ & $2(3)$ \\
\hline C19 & $21(3)$ & $29(3)$ & $18(3)$ & $1(2)$ & $3(2)$ & $5(3)$ \\
\hline C20 & $16(3)$ & $35(3)$ & $22(3)$ & $1(3)$ & $3(2)$ & $5(3)$ \\
\hline C21 & $20(3)$ & $49(4)$ & $27(3)$ & $-5(3)$ & $2(2)$ & $8(3)$ \\
\hline $\mathrm{C} 22$ & $30(3)$ & $31(4)$ & $25(3)$ & $-10(3)$ & $2(2)$ & $7(3)$ \\
\hline $\mathrm{C} 23$ & $25(3)$ & $31(4)$ & $23(3)$ & $-5(3)$ & $1(2)$ & $1(3)$ \\
\hline C24 & $16(3)$ & $24(3)$ & $19(3)$ & $0(2)$ & $1(2)$ & $3(2)$ \\
\hline $\mathrm{C} 25$ & $21(3)$ & $18(3)$ & $18(3)$ & $-1(2)$ & $3(2)$ & $-1(2)$ \\
\hline C26 & $24(3)$ & $18(3)$ & $27(3)$ & $3(2)$ & $0(2)$ & $0(2)$ \\
\hline $\mathrm{C} 27$ & $37(4)$ & $20(3)$ & $26(3)$ & $3(2)$ & $-2(3)$ & $-6(3)$ \\
\hline C28 & $33(4)$ & $24(3)$ & $35(3)$ & $4(3)$ & $5(3)$ & $-12(3)$ \\
\hline C29 & $25(3)$ & $26(3)$ & $36(3)$ & $6(3)$ & $3(2)$ & $-3(3)$ \\
\hline C30 & $24(3)$ & $19(3)$ & $22(3)$ & $0(2)$ & $-1(2)$ & $-2(2)$ \\
\hline C31 & $18(3)$ & $16(3)$ & $19(2)$ & $-4(2)$ & $0(2)$ & $-5(2)$ \\
\hline C32 & $14(3)$ & $20(3)$ & $27(3)$ & $1(2)$ & $1(2)$ & $0(2)$ \\
\hline C33 & $18(3)$ & $27(3)$ & $29(3)$ & $-5(3)$ & $3(2)$ & $-4(2)$ \\
\hline C34 & $31(3)$ & $31(4)$ & $23(3)$ & $-1(3)$ & $-3(3)$ & $1(3)$ \\
\hline C35 & $39(4)$ & $31(4)$ & $32(3)$ & $11(3)$ & $4(3)$ & $0(3)$ \\
\hline
\end{tabular}




\begin{tabular}{|c|c|c|c|c|c|c|}
\hline C36 & $28(3)$ & $17(3)$ & $38(3)$ & $7(3)$ & $-5(3)$ & $-3(3)$ \\
\hline C37 & $17(3)$ & $20(3)$ & $25(3)$ & $-2(2)$ & $2(2)$ & $-1(2)$ \\
\hline C38 & $32(4)$ & $28(3)$ & $27(3)$ & $-10(3)$ & $15(3)$ & $-12(3)$ \\
\hline C39 & $47(4)$ & $25(3)$ & $27(3)$ & $-4(3)$ & $14(3)$ & $-7(3)$ \\
\hline $\mathrm{C} 40$ & $94(8)$ & $43(5)$ & $38(4)$ & $-2(4)$ & $25(5)$ & $-23(5)$ \\
\hline C41 & $120(10)$ & $78(8)$ & $46(5)$ & $-15(5)$ & $41(6)$ & $-56(7)$ \\
\hline $\mathrm{C} 42$ & $70(7)$ & $108(10)$ & $70(7)$ & $-36(6)$ & $42(6)$ & $-60(7)$ \\
\hline $\mathrm{C} 43$ & $33(4)$ & $72(6)$ & $46(4)$ & $-26(4)$ & $18(3)$ & $-26(4)$ \\
\hline N44 & $31(3)$ & $23(3)$ & $29(3)$ & $9(2)$ & $5(2)$ & $10(2)$ \\
\hline $\mathrm{C} 45$ & $18(3)$ & $9(2)$ & $18(2)$ & $0(2)$ & $1.5(19)$ & $3(2)$ \\
\hline $\mathrm{C} 46$ & $21(3)$ & $21(3)$ & $22(3)$ & $1(2)$ & $3(2)$ & $-6(2)$ \\
\hline $\mathrm{C} 47$ & $14(3)$ & $21(3)$ & $23(3)$ & $1(2)$ & $2(2)$ & $-4(2)$ \\
\hline $\mathrm{C} 48$ & $15(3)$ & $27(3)$ & $33(3)$ & $12(3)$ & $0(2)$ & $-2(3)$ \\
\hline C49 & $22(3)$ & $44(4)$ & $28(3)$ & $11(3)$ & $-4(2)$ & $-13(3)$ \\
\hline C50 & $37(4)$ & $45(4)$ & $22(3)$ & $1(3)$ & $1(3)$ & $-17(3)$ \\
\hline C51 & $29(3)$ & $29(3)$ & $29(3)$ & $-4(3)$ & $6(2)$ & $-7(3)$ \\
\hline C52 & $17(3)$ & $17(3)$ & $28(3)$ & $3(2)$ & $2(2)$ & $2(2)$ \\
\hline C53 & $40(4)$ & $26(4)$ & $30(3)$ & $5(3)$ & $5(3)$ & $10(3)$ \\
\hline C54 & $54(5)$ & $27(4)$ & $38(4)$ & $-4(3)$ & $12(3)$ & $14(3)$ \\
\hline C55 & $41(4)$ & $28(4)$ & $31(3)$ & $-4(3)$ & $9(3)$ & $4(3)$ \\
\hline C56 & $30(3)$ & $28(4)$ & $24(3)$ & $2(3)$ & $6(2)$ & $1(3)$ \\
\hline C57 & $17(3)$ & $19(3)$ & $27(3)$ & $1(2)$ & $2(2)$ & $-1(2)$ \\
\hline C58 & $26(3)$ & 11(3) & $31(3)$ & $0(2)$ & $9(2)$ & $3(2)$ \\
\hline C59 & $31(3)$ & $21(3)$ & $27(3)$ & $6(2)$ & $4(2)$ & $3(3)$ \\
\hline C60 & $30(3)$ & $21(3)$ & $39(3)$ & $7(3)$ & $-4(3)$ & $3(3)$ \\
\hline C61 & $23(3)$ & $17(3)$ & $46(4)$ & $1(3)$ & $-8(3)$ & $7(3)$ \\
\hline C62 & $23(3)$ & $19(3)$ & $37(3)$ & $-7(3)$ & $0(2)$ & $6(2)$ \\
\hline C63 & $11(2)$ & $15(3)$ & $29(3)$ & $-1(2)$ & $1(2)$ & $-3(2)$ \\
\hline C64 & $17(3)$ & $14(3)$ & $25(3)$ & $-2(2)$ & $-1(2)$ & $0(2)$ \\
\hline O5S_1 & $104(5)$ & $126(9)$ & $94(5)$ & $0(5)$ & $13(4)$ & $10(6)$ \\
\hline N1S_1 & $91(3)$ & $90(4)$ & $96(3)$ & $-4(2)$ & $14(2)$ & $-3(2)$ \\
\hline C2S_1 & $110(6)$ & 161(18) & $130(6)$ & $54(10)$ & $-11(6)$ & $-53(9)$ \\
\hline C3S_1 & $92(4)$ & $97(12)$ & $100(8)$ & $-3(7)$ & $12(3)$ & $-7(5)$ \\
\hline C4S_1 & $95(5)$ & $105(10)$ & $97(4)$ & $2(5)$ & $12(3)$ & $-7(6)$ \\
\hline O5S_2 & $104(5)$ & $126(9)$ & $94(5)$ & $0(5)$ & $13(4)$ & $10(6)$ \\
\hline N1S_2 & $91(3)$ & $90(4)$ & $96(3)$ & $-4(2)$ & $14(2)$ & $-3(2)$ \\
\hline C2S_2 & $110(6)$ & 161(18) & $130(6)$ & $54(10)$ & $-11(6)$ & $-53(9)$ \\
\hline C3S_2 & $92(4)$ & $97(12)$ & $100(8)$ & $-3(7)$ & $12(3)$ & $-7(5)$ \\
\hline C4S_2 & $95(5)$ & $105(10)$ & $97(4)$ & $2(5)$ & $12(3)$ & $-7(6)$ \\
\hline
\end{tabular}


Table S8. Bond Lengths for GT-18.

\begin{tabular}{|c|c|c|c|c|c|}
\hline Atom & Atom & Length/Å & Atom & Atom & Length/Å \\
\hline $\mathrm{Zn} 1$ & N1 & $2.008(5)$ & $\mathrm{C} 4$ & $\mathrm{C} 5$ & $1.396(8)$ \\
\hline $\mathrm{Zn} 1$ & $\mathrm{~N} 2$ & $2.006(5)$ & $\mathrm{C} 5$ & C64 & $1.406(8)$ \\
\hline $\mathrm{Zn} 1$ & N5 & $2.018(5)$ & C6 & $\mathrm{C} 7$ & $1.408(8)$ \\
\hline $\mathrm{Zn} 1$ & N24 & $2.004(5)$ & C6 & C11 & $1.407(8)$ \\
\hline $\mathrm{Zn} 2$ & N7 & $2.012(5)$ & $\mathrm{C} 7$ & $\mathrm{C} 8$ & $1.362(9)$ \\
\hline $\mathrm{Zn} 2$ & N8 & $1.992(5)$ & $\mathrm{C} 8$ & C9 & $1.425(9)$ \\
\hline $\mathrm{Zn} 2$ & N15 & $1.997(6)$ & C9 & $\mathrm{C} 10$ & $1.364(9)$ \\
\hline $\mathrm{Zn} 2$ & N21 & $1.989(5)$ & $\mathrm{C} 10$ & $\mathrm{C} 11$ & $1.411(9)$ \\
\hline $\mathrm{Zn} 3$ & N10 & $1.992(5)$ & $\mathrm{C} 13$ & $\mathrm{C} 14$ & $1.396(10)$ \\
\hline $\mathrm{Zn} 3$ & N11 & $2.009(5)$ & C13 & C18 & $1.394(10)$ \\
\hline $\mathrm{Zn} 3$ & N16 & $2.008(5)$ & C14 & C15 & $1.356(15)$ \\
\hline $\mathrm{Zn} 3$ & N19 & $1.986(5)$ & $\mathrm{C} 15$ & $\mathrm{C} 16$ & $1.401(16)$ \\
\hline $\mathrm{Zn} 4$ & N13 & $2.009(5)$ & $\mathrm{C} 16$ & $\mathrm{C} 17$ & $1.383(12)$ \\
\hline $\mathrm{Zn} 4$ & N14 & $2.019(5)$ & $\mathrm{C} 17$ & C18 & $1.397(10)$ \\
\hline $\mathrm{Zn} 4$ & N181 & $1.992(5)$ & C19 & $\mathrm{C} 20$ & $1.410(8)$ \\
\hline Zn4 & N202 & $1.990(5)$ & C19 & $\mathrm{C} 24$ & $1.401(8)$ \\
\hline Zn5 & N43 & $1.994(5)$ & $\mathrm{C} 20$ & $\mathrm{C} 21$ & $1.359(9)$ \\
\hline $\mathrm{Zn} 5$ & $\mathrm{~N} 22$ & $2.026(5)$ & $\mathrm{C} 21$ & $\mathrm{C} 22$ & $1.402(10)$ \\
\hline $\mathrm{Zn} 5$ & N23 & $1.997(5)$ & $\mathrm{C} 22$ & $\mathrm{C} 23$ & $1.373(9)$ \\
\hline Zn5 & N264 & $2.002(5)$ & $\mathrm{C} 23$ & $\mathrm{C} 24$ & $1.395(8)$ \\
\hline N1 & N25 & $1.335(7)$ & $\mathrm{C} 25$ & $\mathrm{C} 26$ & $1.401(8)$ \\
\hline N1 & $\mathrm{C} 5$ & $1.371(8)$ & $\mathrm{C} 25$ & C30 & $1.408(9)$ \\
\hline $\mathrm{N} 2$ & N3 & $1.333(7)$ & $\mathrm{C} 26$ & $\mathrm{C} 27$ & $1.383(9)$ \\
\hline N2 & $\mathrm{C} 11$ & $1.363(7)$ & $\mathrm{C} 27$ & $\mathrm{C} 28$ & $1.391(10)$ \\
\hline N3 & N4 & $1.340(7)$ & $\mathrm{C} 28$ & $\mathrm{C} 29$ & $1.375(9)$ \\
\hline N4 & C6 & $1.367(7)$ & C29 & C30 & $1.392(9)$ \\
\hline N5 & N6 & $1.316(7)$ & C32 & C33 & $1.396(8)$ \\
\hline N5 & C63 & $1.372(7)$ & C32 & C37 & $1.393(8)$ \\
\hline N6 & N7 & $1.324(7)$ & C33 & C34 & $1.379(9)$ \\
\hline N7 & C58 & $1.379(7)$ & C34 & C35 & $1.429(10)$ \\
\hline N8 & N9 & $1.334(7)$ & C35 & C36 & $1.354(9)$ \\
\hline N8 & C39 & $1.379(9)$ & C36 & C37 & $1.398(8)$ \\
\hline N9 & N10 & $1.328(7)$ & C38 & C39 & $1.400(10)$ \\
\hline N10 & C38 & $1.379(8)$ & C38 & $\mathrm{C} 43$ & $1.411(10)$ \\
\hline N11 & N12 & $1.339(7)$ & C39 & $\mathrm{C} 40$ & $1.396(10)$ \\
\hline N11 & $\mathrm{C} 24$ & $1.390(7)$ & $\mathrm{C} 40$ & C41 & $1.373(16)$ \\
\hline N12 & N13 & $1.325(7)$ & C41 & $\mathrm{C} 42$ & $1.425(18)$ \\
\hline N13 & C19 & $1.378(8)$ & $\mathrm{C} 42$ & $\mathrm{C} 43$ & $1.377(13)$ \\
\hline N14 & $\mathrm{C} 12$ & $1.320(7)$ & $\mathrm{C} 46$ & $\mathrm{C} 47$ & $1.389(9)$ \\
\hline
\end{tabular}




\begin{tabular}{|c|c|c|c|c|c|}
\hline N14 & C18 & $1.365(8)$ & $\mathrm{C} 46$ & C51 & $1.404(8)$ \\
\hline N15 & $\mathrm{C} 12$ & $1.333(7)$ & $\mathrm{C} 47$ & $\mathrm{C} 48$ & $1.407(8)$ \\
\hline N15 & $\mathrm{C} 13$ & $1.380(9)$ & $\mathrm{C} 48$ & C49 & $1.365(10)$ \\
\hline N16 & N17 & $1.335(7)$ & C49 & $\mathrm{C} 50$ & $1.396(11)$ \\
\hline N16 & C30 & $1.362(8)$ & C50 & C51 & $1.369(10)$ \\
\hline N17 & N18 & $1.341(7)$ & C52 & C53 & $1.386(9)$ \\
\hline N18 & $\mathrm{C} 25$ & $1.361(7)$ & C52 & C57 & $1.400(8)$ \\
\hline N19 & C31 & $1.337(8)$ & C53 & C54 & $1.375(9)$ \\
\hline N19 & C37 & $1.380(7)$ & C54 & $\mathrm{C} 55$ & $1.393(10)$ \\
\hline $\mathrm{N} 20$ & C31 & $1.333(7)$ & C55 & C56 & $1.380(9)$ \\
\hline $\mathrm{N} 20$ & C32 & $1.393(8)$ & C56 & C57 & $1.404(8)$ \\
\hline $\mathrm{N} 21$ & N44 & $1.335(7)$ & C58 & C59 & $1.404(8)$ \\
\hline N21 & C57 & $1.376(8)$ & C58 & C63 & $1.383(8)$ \\
\hline $\mathrm{N} 22$ & N44 & $1.336(7)$ & C59 & C60 & $1.384(9)$ \\
\hline N22 & C52 & $1.373(8)$ & C60 & C61 & $1.397(10)$ \\
\hline $\mathrm{N} 23$ & $\mathrm{C} 45$ & $1.326(7)$ & C61 & C62 & $1.369(9)$ \\
\hline $\mathrm{N} 23$ & $\mathrm{C} 47$ & $1.366(8)$ & C62 & C63 & $1.414(8)$ \\
\hline $\mathrm{N} 24$ & $\mathrm{C} 45$ & $1.333(7)$ & O5S_1 & C4S_1 & $1.234(7)$ \\
\hline N24 & C46 & $1.389(7)$ & N1S_1 & C2S_1 & $1.449(8)$ \\
\hline $\mathrm{N} 25$ & N26 & $1.327(7)$ & N1S_1 & C3S_1 & $1.437(8)$ \\
\hline N26 & C64 & $1.364(7)$ & N1S_1 & C4S_1 & $1.381(19)$ \\
\hline $\mathrm{C} 1$ & $\mathrm{C} 2$ & $1.371(9)$ & O5S_2 & C4S_2 & $1.232(6)$ \\
\hline $\mathrm{C} 1$ & C64 & $1.404(8)$ & N1S_2 & C2S_2 & $1.449(9)$ \\
\hline $\mathrm{C} 2$ & $\mathrm{C} 3$ & $1.422(10)$ & N1S_2 & C3S_2 & $1.437(9)$ \\
\hline C3 & $\mathrm{C} 4$ & $1.367(9)$ & N1S_2 & $\mathrm{C} 4 \mathrm{~S}_{-} 2$ & $1.381(19)$ \\
\hline
\end{tabular}

Table S9. Bond Angles for GT-18.

\begin{tabular}{|c|c|c|c|c|c|c|c|}
\hline Atom & Atom & Atom & Angle $/^{\circ}$ & Atom & Atom & Atom & Angle $/^{\circ}$ \\
\hline N1 & $\mathrm{Zn} 1$ & N5 & $105.1(2)$ & N4 & C6 & $\mathrm{C} 11$ & $106.1(5)$ \\
\hline $\mathrm{N} 2$ & $\mathrm{Zn} 1$ & N1 & $114.1(2)$ & C11 & C6 & $\mathrm{C} 7$ & $121.7(5)$ \\
\hline N2 & $\mathrm{Zn} 1$ & N5 & $105.7(2)$ & $\mathrm{C} 8$ & $\mathrm{C} 7$ & C6 & $116.2(6)$ \\
\hline $\mathrm{N} 24$ & $\mathrm{Zn} 1$ & N1 & $109.8(2)$ & $\mathrm{C} 7$ & $\mathrm{C} 8$ & C9 & $122.8(6)$ \\
\hline $\mathrm{N} 24$ & $\mathrm{Zn} 1$ & $\mathrm{~N} 2$ & $112.2(2)$ & $\mathrm{C} 10$ & C9 & $\mathrm{C} 8$ & $121.3(6)$ \\
\hline $\mathrm{N} 24$ & $\mathrm{Zn} 1$ & N5 & 109.61(19) & C9 & $\mathrm{C} 10$ & $\mathrm{C} 11$ & $117.2(6)$ \\
\hline N8 & $\mathrm{Zn} 2$ & N7 & $104.5(2)$ & $\mathrm{N} 2$ & $\mathrm{C} 11$ & C6 & $106.9(5)$ \\
\hline N8 & $\mathrm{Zn} 2$ & N15 & $108.6(2)$ & $\mathrm{N} 2$ & $\mathrm{C} 11$ & $\mathrm{C} 10$ & $132.3(5)$ \\
\hline N15 & $\mathrm{Zn} 2$ & N7 & $104.8(2)$ & C6 & C11 & $\mathrm{C} 10$ & $120.8(5)$ \\
\hline $\mathrm{N} 21$ & $\mathrm{Zn} 2$ & N7 & $109.8(2)$ & N14 & $\mathrm{C} 12$ & N15 & $112.0(5)$ \\
\hline N21 & $\mathrm{Zn} 2$ & N8 & $114.6(2)$ & N15 & C13 & $\mathrm{C} 14$ & $132.1(8)$ \\
\hline $\mathrm{N} 21$ & $\mathrm{Zn} 2$ & N15 & $113.7(2)$ & N15 & C13 & $\mathrm{C} 18$ & $107.0(6)$ \\
\hline
\end{tabular}




\begin{tabular}{|c|c|c|c|c|c|c|c|}
\hline N10 & Zn3 & N11 & $107.8(2)$ & $\mathrm{C} 18$ & $\mathrm{C} 13$ & $\mathrm{C} 14$ & $120.9(7)$ \\
\hline N10 & Zn3 & N16 & $110.2(2)$ & $\mathrm{C} 15$ & $\mathrm{C} 14$ & $\mathrm{C} 13$ & $117.9(9)$ \\
\hline N16 & Zn3 & N11 & 109.2(2) & $\mathrm{C} 14$ & $\mathrm{C} 15$ & $\mathrm{C} 16$ & $121.4(8)$ \\
\hline N19 & $\mathrm{Zn} 3$ & N10 & $109.6(2)$ & $\mathrm{C} 17$ & $\mathrm{C} 16$ & $\mathrm{C} 15$ & $121.7(9)$ \\
\hline N19 & Zn3 & N11 & $110.8(2)$ & $\mathrm{C} 16$ & $\mathrm{C} 17$ & $\mathrm{C} 18$ & $116.7(9)$ \\
\hline N19 & Zn3 & N16 & $109.1(2)$ & N14 & $\mathrm{C} 18$ & $\mathrm{C} 13$ & $107.1(6)$ \\
\hline N13 & Zn4 & N14 & $103.5(2)$ & N14 & $\mathrm{C} 18$ & $\mathrm{C} 17$ & $131.6(7)$ \\
\hline N181 & $\mathrm{Zn} 4$ & N13 & $112.3(2)$ & $\mathrm{C} 13$ & $\mathrm{C} 18$ & $\mathrm{C} 17$ & $121.3(6)$ \\
\hline N181 & $\mathrm{Zn} 4$ & N14 & $102.4(2)$ & N13 & C19 & $\mathrm{C} 20$ & $131.5(6)$ \\
\hline N202 & $\mathrm{Zn} 4$ & N13 & $114.1(2)$ & N13 & C19 & $\mathrm{C} 24$ & $106.9(5)$ \\
\hline N202 & Zn4 & N14 & $108.9(2)$ & $\mathrm{C} 24$ & C19 & $\mathrm{C} 20$ & $121.5(6)$ \\
\hline N202 & $\mathrm{Zn} 4$ & N181 & $114.3(2)$ & $\mathrm{C} 21$ & $\mathrm{C} 20$ & C19 & $116.2(6)$ \\
\hline N43 & Zn5 & N22 & $107.1(2)$ & $\mathrm{C} 20$ & $\mathrm{C} 21$ & $\mathrm{C} 22$ & $122.4(6)$ \\
\hline N43 & Zn5 & $\mathrm{N} 23$ & $109.1(2)$ & $\mathrm{C} 23$ & $\mathrm{C} 22$ & $\mathrm{C} 21$ & $122.1(6)$ \\
\hline N43 & Zn5 & N264 & $115.1(2)$ & $\mathrm{C} 22$ & $\mathrm{C} 23$ & $\mathrm{C} 24$ & $116.5(6)$ \\
\hline $\mathrm{N} 23$ & Zn5 & N22 & 109.71(19) & N11 & $\mathrm{C} 24$ & $\mathrm{C} 19$ & $106.3(5)$ \\
\hline $\mathrm{N} 23$ & Zn5 & N264 & $114.0(2)$ & N11 & $\mathrm{C} 24$ & $\mathrm{C} 23$ & $132.5(6)$ \\
\hline N264 & Zn5 & N22 & $101.23(19)$ & $\mathrm{C} 23$ & $\mathrm{C} 24$ & $\mathrm{C} 19$ & $121.2(5)$ \\
\hline $\mathrm{N} 25$ & N1 & $\mathrm{Zn} 1$ & $115.9(4)$ & N18 & $\mathrm{C} 25$ & $\mathrm{C} 26$ & $131.9(6)$ \\
\hline $\mathrm{N} 25$ & N1 & $\mathrm{C} 5$ & $108.6(5)$ & N18 & $\mathrm{C} 25$ & $\mathrm{C} 30$ & $106.9(5)$ \\
\hline $\mathrm{C} 5$ & N1 & $\mathrm{Zn} 1$ & $135.2(4)$ & $\mathrm{C} 26$ & $\mathrm{C} 25$ & $\mathrm{C} 30$ & $121.1(6)$ \\
\hline N3 & $\mathrm{N} 2$ & $\mathrm{Zn} 1$ & $117.0(4)$ & $\mathrm{C} 27$ & $\mathrm{C} 26$ & $\mathrm{C} 25$ & $116.5(6)$ \\
\hline N3 & $\mathrm{N} 2$ & $\mathrm{C} 11$ & $108.7(5)$ & $\mathrm{C} 26$ & $\mathrm{C} 27$ & $\mathrm{C} 28$ & $121.7(6)$ \\
\hline C11 & $\mathrm{N} 2$ & $\mathrm{Zn} 1$ & $133.5(4)$ & C29 & $\mathrm{C} 28$ & $\mathrm{C} 27$ & $122.6(6)$ \\
\hline N2 & $\mathrm{N} 3$ & N4 & $109.5(5)$ & $\mathrm{C} 28$ & $\mathrm{C} 29$ & $\mathrm{C} 30$ & $116.6(6)$ \\
\hline N3 & N4 & Zn55 & $115.2(4)$ & N16 & $\mathrm{C} 30$ & $\mathrm{C} 25$ & $106.4(5)$ \\
\hline N3 & N4 & C6 & $108.8(5)$ & N16 & $\mathrm{C} 30$ & $\mathrm{C} 29$ & $132.2(6)$ \\
\hline C6 & N4 & Zn55 & $131.7(4)$ & C29 & $\mathrm{C} 30$ & $\mathrm{C} 25$ & $121.5(6)$ \\
\hline N6 & N5 & $\mathrm{Zn} 1$ & $117.7(4)$ & $\mathrm{N} 20$ & C31 & N19 & $113.5(5)$ \\
\hline N6 & N5 & C63 & $108.0(5)$ & $\mathrm{N} 20$ & C32 & C33 & $132.0(6)$ \\
\hline C63 & N5 & $\mathrm{Zn} 1$ & $133.3(4)$ & $\mathrm{C} 37$ & C32 & N20 & $107.3(5)$ \\
\hline N5 & N6 & N7 & $110.7(5)$ & C37 & C32 & C33 & $120.7(6)$ \\
\hline N6 & N7 & $\mathrm{Zn} 2$ & $123.3(4)$ & $\mathrm{C} 34$ & C33 & C32 & $117.7(6)$ \\
\hline N6 & N7 & C58 & $107.8(5)$ & $\mathrm{C} 33$ & C34 & $\mathrm{C} 35$ & $120.8(6)$ \\
\hline C58 & N7 & $\mathrm{Zn} 2$ & $128.7(4)$ & $\mathrm{C} 36$ & C35 & C34 & $121.2(6)$ \\
\hline N9 & N8 & $\mathrm{Zn} 2$ & $122.9(4)$ & $\mathrm{C} 35$ & C36 & C37 & $117.9(6)$ \\
\hline N9 & N8 & C39 & $106.5(5)$ & N19 & C37 & C32 & $108.0(5)$ \\
\hline C39 & N8 & $\mathrm{Zn} 2$ & $130.4(4)$ & N19 & C37 & C36 & $130.4(6)$ \\
\hline N10 & N9 & N8 & $112.2(5)$ & C32 & C37 & C36 & $121.6(5)$ \\
\hline N9 & N10 & $\mathrm{Zn} 3$ & $118.9(4)$ & N10 & C38 & C39 & $106.8(6)$ \\
\hline N9 & N10 & C38 & $107.1(5)$ & N10 & C38 & $\mathrm{C} 43$ & $130.9(7)$ \\
\hline C38 & N10 & Zn3 & $133.3(4)$ & C39 & C38 & $\mathrm{C} 43$ & $122.3(7)$ \\
\hline N12 & N11 & $\mathrm{Zn} 3$ & $115.3(4)$ & N8 & C39 & C38 & $107.4(5)$ \\
\hline
\end{tabular}




\begin{tabular}{|c|c|c|c|c|c|c|c|}
\hline N12 & N11 & $\mathrm{C} 24$ & $107.8(5)$ & N8 & C39 & $\mathrm{C} 40$ & $130.4(8)$ \\
\hline $\mathrm{C} 24$ & N11 & Zn3 & $135.5(4)$ & $\mathrm{C} 40$ & C39 & $\mathrm{C} 38$ & $122.3(8)$ \\
\hline N13 & N12 & N11 & $110.7(5)$ & $\mathrm{C} 41$ & C40 & C39 & $115.3(9)$ \\
\hline N12 & N13 & $\mathrm{Zn} 4$ & $116.1(4)$ & $\mathrm{C} 40$ & $\mathrm{C} 41$ & $\mathrm{C} 42$ & $123.0(9)$ \\
\hline N12 & N13 & C19 & $108.4(5)$ & $\mathrm{C} 43$ & $\mathrm{C} 42$ & $\mathrm{C} 41$ & $121.8(9)$ \\
\hline C19 & N13 & $\mathrm{Zn} 4$ & $134.2(4)$ & $\mathrm{C} 42$ & C43 & $\mathrm{C} 38$ & $115.2(9)$ \\
\hline C12 & N14 & $\mathrm{Zn} 4$ & $118.0(4)$ & N21 & N44 & N22 & $111.5(5)$ \\
\hline C12 & N14 & $\mathrm{C} 18$ & $107.4(5)$ & N23 & $\mathrm{C} 45$ & N24 & $113.2(5)$ \\
\hline C18 & N14 & $\mathrm{Zn} 4$ & $133.8(4)$ & N24 & C46 & $\mathrm{C} 51$ & $130.8(6)$ \\
\hline C12 & N15 & $\mathrm{Zn} 2$ & $120.7(4)$ & $\mathrm{C} 47$ & C46 & N24 & $107.2(5)$ \\
\hline C12 & N15 & $\mathrm{C} 13$ & $106.4(5)$ & $\mathrm{C} 47$ & C46 & $\mathrm{C} 51$ & $122.1(6)$ \\
\hline C13 & N15 & $\mathrm{Zn} 2$ & $132.3(5)$ & $\mathrm{N} 23$ & C47 & $\mathrm{C} 46$ & $107.9(5)$ \\
\hline N17 & N16 & $\mathrm{Zn} 3$ & $114.4(4)$ & $\mathrm{N} 23$ & $\mathrm{C} 47$ & $\mathrm{C} 48$ & $131.4(6)$ \\
\hline N17 & N16 & C30 & $108.7(5)$ & $\mathrm{C} 46$ & C47 & $\mathrm{C} 48$ & $120.7(6)$ \\
\hline $\mathrm{C} 30$ & N16 & $\mathrm{Zn} 3$ & $136.5(4)$ & $\mathrm{C} 49$ & C48 & $\mathrm{C} 47$ & $116.9(6)$ \\
\hline N16 & N17 & N18 & $109.7(5)$ & $\mathrm{C} 48$ & C49 & $\mathrm{C} 50$ & $121.9(6)$ \\
\hline N17 & N18 & $\mathrm{Zn} 46$ & $115.4(4)$ & C51 & C50 & $\mathrm{C} 49$ & $122.5(6)$ \\
\hline N17 & N18 & $\mathrm{C} 25$ & $108.2(5)$ & $\mathrm{C} 50$ & C51 & $\mathrm{C} 46$ & $115.9(7)$ \\
\hline $\mathrm{C} 25$ & N18 & $\mathrm{Zn} 46$ & $135.3(4)$ & N22 & $\mathrm{C} 52$ & C53 & $132.3(5)$ \\
\hline C31 & N19 & Zn3 & $127.3(4)$ & N22 & $\mathrm{C} 52$ & C57 & $106.7(5)$ \\
\hline C31 & N19 & C37 & $105.7(5)$ & $\mathrm{C} 53$ & $\mathrm{C} 52$ & C57 & $121.0(6)$ \\
\hline C37 & N19 & $\mathrm{Zn} 3$ & $127.0(4)$ & $\mathrm{C} 54$ & C53 & C52 & $118.0(6)$ \\
\hline C31 & N20 & $\mathrm{Zn} 47$ & $124.3(4)$ & C53 & C54 & C55 & $120.9(7)$ \\
\hline C31 & N20 & C32 & $105.6(5)$ & C56 & $\mathrm{C} 55$ & C54 & $122.6(6)$ \\
\hline C32 & N20 & $\mathrm{Zn} 47$ & $130.0(4)$ & $\mathrm{C} 55$ & $\mathrm{C} 56$ & C57 & $116.2(6)$ \\
\hline N44 & N21 & $\mathrm{Zn} 2$ & $119.5(4)$ & N21 & $\mathrm{C} 57$ & C52 & $107.5(5)$ \\
\hline N44 & N21 & C57 & $106.8(5)$ & N21 & C57 & C56 & $131.2(6)$ \\
\hline $\mathrm{C} 57$ & $\mathrm{~N} 21$ & $\mathrm{Zn} 2$ & $132.8(4)$ & $\mathrm{C} 52$ & C57 & C56 & $121.3(6)$ \\
\hline N44 & $\mathrm{N} 22$ & $\mathrm{Zn} 5$ & $121.0(4)$ & N7 & C58 & C59 & $131.1(6)$ \\
\hline N44 & N22 & C52 & $107.4(5)$ & N7 & $\mathrm{C} 58$ & C63 & $106.4(5)$ \\
\hline C52 & N22 & $\mathrm{Zn} 5$ & $131.5(4)$ & C63 & C58 & C59 & $122.6(5)$ \\
\hline $\mathrm{C} 45$ & $\mathrm{~N} 23$ & $\mathrm{Zn} 5$ & $120.9(4)$ & C60 & C59 & C58 & $115.7(6)$ \\
\hline $\mathrm{C} 45$ & $\mathrm{~N} 23$ & $\mathrm{C} 47$ & $106.3(5)$ & C59 & C60 & C61 & $121.7(6)$ \\
\hline $\mathrm{C} 47$ & $\mathrm{~N} 23$ & $\mathrm{Zn} 5$ & $132.4(4)$ & C62 & C61 & C60 & $122.9(6)$ \\
\hline $\mathrm{C} 45$ & N24 & $\mathrm{Zn} 1$ & $123.1(4)$ & C61 & C62 & C63 & $116.0(6)$ \\
\hline $\mathrm{C} 45$ & N24 & $\mathrm{C} 46$ & $105.4(5)$ & N5 & C63 & C58 & $107.0(5)$ \\
\hline $\mathrm{C} 46$ & N24 & $\mathrm{Zn} 1$ & $131.5(4)$ & N5 & C63 & C62 & $131.9(6)$ \\
\hline N26 & N25 & N1 & $109.8(5)$ & C58 & C63 & C62 & $121.1(5)$ \\
\hline $\mathrm{N} 25$ & N26 & Zn58 & $124.3(4)$ & N26 & C64 & $\mathrm{C} 1$ & $131.9(5)$ \\
\hline $\mathrm{N} 25$ & N26 & C64 & $108.8(5)$ & N26 & C64 & $\mathrm{C} 5$ & $106.7(5)$ \\
\hline C64 & N26 & Zn58 & $126.1(4)$ & $\mathrm{C} 1$ & C64 & $\mathrm{C} 5$ & $121.4(5)$ \\
\hline $\mathrm{C} 2$ & $\mathrm{C} 1$ & C64 & $116.5(6)$ & C3S_1 & N1S_1 & $\mathrm{C} 2 \mathrm{~S} \_1$ & $116.7(14)$ \\
\hline $\mathrm{C} 1$ & $\mathrm{C} 2$ & C3 & $122.4(6)$ & $\mathrm{C} 4 \mathrm{~S} \_1$ & N1S_1 & C2S_1 & $131.7(13)$ \\
\hline
\end{tabular}




\begin{tabular}{|c|c|c|c|c|c|c|c|}
\hline $\mathrm{C} 4$ & $\mathrm{C} 3$ & $\mathrm{C} 2$ & $120.7(6)$ & C4S_1 & N1S_1 & C3S_1 & $111.6(13)$ \\
\hline $\mathrm{C} 3$ & $\mathrm{C} 4$ & $\mathrm{C} 5$ & $118.1(6)$ & O5S_1 & C4S_1 & N1S_1 & $135.2(18)$ \\
\hline N1 & $\mathrm{C} 5$ & $\mathrm{C} 4$ & $133.0(5)$ & C3S_2 & N1S_2 & C2S_2 & $116.6(15)$ \\
\hline N1 & $\mathrm{C} 5$ & C64 & $106.1(5)$ & C4S_2 & N1S_2 & C2S_2 & $131.5(14)$ \\
\hline $\mathrm{C} 4$ & C5 & C64 & $120.9(5)$ & C4S_2 & N1S_2 & C3S_2 & $111.5(13)$ \\
\hline N4 & $\mathrm{C} 6$ & C7 & $132.2(6)$ & O5S 2 & $\mathrm{C} 4 \mathrm{~S} 2$ & N1S 2 & $135.5(19)$ \\
\hline
\end{tabular}

Table S10. Torsion Angles for GT-18.

\begin{tabular}{|c|c|c|c|c|c|c|c|c|c|}
\hline $\mathbf{A}$ & B & C & D & Angle ${ }^{\circ}$ & A & B & C & D & Angle $/^{\circ}$ \\
\hline Zn1 & N1 & $\mathrm{N} 25$ & $\mathrm{~N} 26$ & $-173.8(4)$ & $\mathrm{N} 25$ & N1 & $\mathrm{C} 5$ & $\mathrm{C} 4$ & $179.5(6)$ \\
\hline Zn1 & N1 & $\mathrm{C} 5$ & $\mathrm{C} 4$ & $-8.4(10)$ & $\mathrm{N} 25$ & N1 & $\mathrm{C} 5$ & C64 & $-0.1(6)$ \\
\hline Zn1 & N1 & $\mathrm{C} 5$ & C64 & $172.0(4)$ & $\mathrm{N} 25$ & $\mathrm{~N} 26$ & C64 & $\mathrm{C} 1$ & $179.9(6)$ \\
\hline Zn1 & $\mathrm{N} 2$ & N3 & N4 & $-170.5(4)$ & $\mathrm{N} 25$ & $\mathrm{~N} 26$ & C64 & $\mathrm{C} 5$ & $0.0(6)$ \\
\hline Zn1 & $\mathrm{N} 2$ & $\mathrm{C} 11$ & C6 & $168.4(4)$ & $\mathrm{C} 1$ & $\mathrm{C} 2$ & C3 & $\mathrm{C} 4$ & $0.6(10)$ \\
\hline Zn1 & $\mathrm{N} 2$ & $\mathrm{C} 11$ & $\mathrm{C} 10$ & $-10.9(10)$ & $\mathrm{C} 2$ & $\mathrm{C} 1$ & C64 & $\mathrm{N} 26$ & $\begin{array}{l}- \\
179.5(6)\end{array}$ \\
\hline Zn1 & N5 & N6 & N7 & $169.5(4)$ & $\mathrm{C} 2$ & $\mathrm{C} 1$ & C64 & $\mathrm{C} 5$ & $0.4(9)$ \\
\hline Zn1 & N5 & C63 & C58 & $-166.5(5)$ & $\mathrm{C} 2$ & $\mathrm{C} 3$ & $\mathrm{C} 4$ & $\mathrm{C} 5$ & $0.3(9)$ \\
\hline Zn1 & N5 & C63 & C62 & $14.3(10)$ & $\mathrm{C} 3$ & $\mathrm{C} 4$ & $\mathrm{C} 5$ & N1 & $179.6(6)$ \\
\hline $\mathrm{Zn} 1$ & $\mathrm{~N} 24$ & $\mathrm{C} 45$ & $\mathrm{~N} 23$ & $176.7(4)$ & $\mathrm{C} 3$ & $\mathrm{C} 4$ & $\mathrm{C} 5$ & C64 & $-0.8(8)$ \\
\hline Zn1 & $\mathrm{N} 24$ & $\mathrm{C} 46$ & $\mathrm{C} 47$ & $-175.4(4)$ & $\mathrm{C} 4$ & $\mathrm{C} 5$ & C64 & $\mathrm{N} 26$ & $\begin{array}{l}- \\
179.6(5)\end{array}$ \\
\hline $\mathrm{Zn} 1$ & $\mathrm{~N} 24$ & $\mathrm{C} 46$ & $\mathrm{C} 51$ & $5.2(9)$ & $\mathrm{C} 4$ & $\mathrm{C} 5$ & C64 & $\mathrm{C} 1$ & $0.5(9)$ \\
\hline $\mathrm{Zn} 2$ & N7 & $\mathrm{C} 58$ & C59 & $5.1(11)$ & $\mathrm{C} 5$ & N1 & $\mathrm{N} 25$ & $\mathrm{~N} 26$ & $0.1(7)$ \\
\hline $\mathrm{Zn} 2$ & N7 & C58 & $\mathrm{C} 63$ & $-175.2(4)$ & C6 & C7 & $\mathrm{C} 8$ & C9 & $1.3(9)$ \\
\hline $\mathrm{Zn} 2$ & N8 & N9 & N10 & $174.6(4)$ & C7 & C6 & $\mathrm{C} 11$ & $\mathrm{~N} 2$ & $\begin{array}{l}- \\
178.5(5)\end{array}$ \\
\hline $\mathrm{Zn} 2$ & N8 & C39 & $\mathrm{C} 38$ & $-173.4(4)$ & C7 & C6 & $\mathrm{C} 11$ & $\mathrm{C} 10$ & $0.8(9)$ \\
\hline $\mathrm{Zn} 2$ & N8 & C39 & $\mathrm{C} 40$ & $5.6(12)$ & C7 & $\mathrm{C} 8$ & C9 & $\mathrm{C} 10$ & $0.7(10)$ \\
\hline $\mathrm{Zn} 2$ & N15 & $\mathrm{C} 12$ & N14 & $-172.8(4)$ & $\mathrm{C} 8$ & C9 & $\mathrm{C} 10$ & $\mathrm{C} 11$ & $-2.0(9)$ \\
\hline $\mathrm{Zn} 2$ & N15 & $\mathrm{C} 13$ & $\mathrm{C} 14$ & $-8.0(13)$ & C9 & $\mathrm{C} 10$ & $\mathrm{C} 11$ & $\mathrm{~N} 2$ & $\begin{array}{l}- \\
179.6(6)\end{array}$ \\
\hline $\mathrm{Zn} 2$ & N15 & $\mathrm{C} 13$ & $\mathrm{C} 18$ & $171.9(5)$ & C9 & $\mathrm{C} 10$ & $\mathrm{C} 11$ & C6 & $1.2(9)$ \\
\hline $\mathrm{Zn} 2$ & $\mathrm{~N} 21$ & N44 & $\mathrm{N} 22$ & $171.0(4)$ & $\mathrm{C} 11$ & $\mathrm{~N} 2$ & N3 & N4 & $0.8(6)$ \\
\hline $\mathrm{Zn} 2$ & $\mathrm{~N} 21$ & C57 & C52 & $-168.4(4)$ & $\mathrm{C} 11$ & C6 & $\mathrm{C} 7$ & $\mathrm{C} 8$ & $-2.1(8)$ \\
\hline $\mathrm{Zn} 2$ & $\mathrm{~N} 21$ & C57 & C56 & $13.0(10)$ & $\mathrm{C} 12$ & N14 & $\mathrm{C} 18$ & $\mathrm{C} 13$ & $0.6(7)$ \\
\hline $\mathrm{Zn} 3$ & N10 & $\mathrm{C} 38$ & C39 & $-168.6(5)$ & $\mathrm{C} 12$ & N14 & $\mathrm{C} 18$ & $\mathrm{C} 17$ & $\begin{array}{l}- \\
179.5(8)\end{array}$ \\
\hline $\mathrm{Zn} 3$ & N10 & $\mathrm{C} 38$ & $\mathrm{C} 43$ & $12.5(11)$ & $\mathrm{C} 12$ & N15 & $\mathrm{C} 13$ & C14 & $\begin{array}{l}- \\
179.3(9)\end{array}$ \\
\hline
\end{tabular}




\begin{tabular}{|c|c|c|c|c|c|c|c|c|c|}
\hline $\mathrm{Zn} 3$ & N11 & N12 & N13 & $-169.2(4)$ & $\mathrm{C} 12$ & N15 & $\mathrm{C} 13$ & $\mathrm{C} 18$ & $0.6(7)$ \\
\hline $\mathrm{Zn} 3$ & N11 & $\mathrm{C} 24$ & $\mathrm{C} 19$ & $165.8(4)$ & $\mathrm{C} 13$ & N15 & $\mathrm{C} 12$ & N14 & $-0.3(7)$ \\
\hline $\mathrm{Zn} 3$ & N11 & $\mathrm{C} 24$ & $\mathrm{C} 23$ & $-12.4(10)$ & $\mathrm{C} 13$ & $\mathrm{C} 14$ & $\mathrm{C} 15$ & $\mathrm{C} 16$ & $2.6(19)$ \\
\hline $\mathrm{Zn} 3$ & N16 & N17 & N18 & $-175.2(4)$ & $\mathrm{C} 14$ & $\mathrm{C} 13$ & C18 & N14 & $179.2(8)$ \\
\hline Zn3 & N16 & C30 & $\mathrm{C} 25$ & $173.6(4)$ & $\mathrm{C} 14$ & $\mathrm{C} 13$ & $\mathrm{C} 18$ & $\mathrm{C} 17$ & $-0.7(12)$ \\
\hline $\mathrm{Zn} 3$ & N16 & C30 & C29 & $-8.2(11)$ & $\mathrm{C} 14$ & $\mathrm{C} 15$ & $\mathrm{C} 16$ & $\mathrm{C} 17$ & $-2.1(19)$ \\
\hline $\mathrm{Zn} 3$ & N19 & C31 & N20 & $177.3(4)$ & $\mathrm{C} 15$ & $\mathrm{C} 16$ & $\mathrm{C} 17$ & $\mathrm{C} 18$ & $0.2(15)$ \\
\hline $\mathrm{Zn} 3$ & N19 & C37 & C32 & $-176.9(4)$ & $\mathrm{C} 16$ & $\mathrm{C} 17$ & C18 & N14 & $\begin{array}{l}- \\
178.7(8)\end{array}$ \\
\hline $\mathrm{Zn} 3$ & N19 & C37 & C36 & $1.1(10)$ & $\mathrm{C} 16$ & $\mathrm{C} 17$ & C18 & $\mathrm{C} 13$ & $1.2(12)$ \\
\hline $\mathrm{Zn} 4$ & N13 & C19 & C20 & $9.9(10)$ & $\mathrm{C} 18$ & N14 & $\mathrm{C} 12$ & N15 & $-0.2(7)$ \\
\hline $\mathrm{Zn} 4$ & N13 & C19 & $\mathrm{C} 24$ & $-166.4(4)$ & $\mathrm{C} 18$ & $\mathrm{C} 13$ & C14 & $\mathrm{C} 15$ & $-1.2(15)$ \\
\hline Zn4 & N14 & $\mathrm{C} 12$ & N15 & $171.2(4)$ & C19 & $\mathrm{C} 20$ & $\mathrm{C} 21$ & $\mathrm{C} 22$ & $0.9(10)$ \\
\hline Zn4 & N14 & $\mathrm{C} 18$ & $\mathrm{C} 13$ & $-168.9(5)$ & $\mathrm{C} 20$ & C19 & C24 & N11 & $\begin{array}{l}- \\
1772(5)\end{array}$ \\
\hline $\mathrm{Zn} 4$ & N14 & $\mathrm{C} 18$ & $\mathrm{C} 17$ & $11.0(12)$ & $\mathrm{C} 20$ & C19 & $\mathrm{C} 24$ & $\mathrm{C} 23$ & $1.3(9)$ \\
\hline Zn41 & N18 & $\mathrm{C} 25$ & C26 & $13.8(10)$ & $\mathrm{C} 20$ & $\mathrm{C} 21$ & $\mathrm{C} 22$ & $\mathrm{C} 23$ & $-1.0(11)$ \\
\hline Zn41 & N18 & $\mathrm{C} 25$ & C30 & $-166.8(4)$ & $\mathrm{C} 21$ & $\mathrm{C} 22$ & $\mathrm{C} 23$ & $\mathrm{C} 24$ & $1.1(10)$ \\
\hline $\mathrm{Zn} 42$ & N20 & C31 & N19 & $175.2(4)$ & $\mathrm{C} 22$ & $\mathrm{C} 23$ & C24 & N11 & $176.7(6)$ \\
\hline $\mathrm{Zn} 42$ & $\mathrm{~N} 20$ & C32 & C33 & $6.9(10)$ & $\mathrm{C} 22$ & $\mathrm{C} 23$ & C24 & C19 & $-1.3(9)$ \\
\hline $\mathrm{Zn} 42$ & N20 & C32 & C37 & $-174.4(4)$ & $\mathrm{C} 24$ & N11 & N12 & N13 & $-0.7(6)$ \\
\hline Zn53 & N4 & C6 & $\mathrm{C} 7$ & $-26.4(10)$ & $\mathrm{C} 24$ & C19 & C20 & $\mathrm{C} 21$ & $-1.1(9)$ \\
\hline Zn53 & N4 & C6 & C11 & $154.5(4)$ & $\mathrm{C} 25$ & $\mathrm{C} 26$ & $\mathrm{C} 27$ & $\mathrm{C} 28$ & $0.2(9)$ \\
\hline $\mathrm{Zn} 5$ & N22 & N44 & N21 & $179.6(4)$ & $\mathrm{C} 26$ & $\mathrm{C} 25$ & C30 & N16 & $178.1(5)$ \\
\hline $\mathrm{Zn} 5$ & N22 & C52 & C53 & $-0.2(10)$ & $\mathrm{C} 26$ & $\mathrm{C} 25$ & C30 & $\mathrm{C} 29$ & $-0.4(9)$ \\
\hline $\mathrm{Zn} 5$ & N22 & C52 & C57 & $-179.4(4)$ & $\mathrm{C} 26$ & $\mathrm{C} 27$ & C28 & C29 & $-0.4(10)$ \\
\hline $\mathrm{Zn} 5$ & $\mathrm{~N} 23$ & $\mathrm{C} 45$ & N24 & $172.8(4)$ & $\mathrm{C} 27$ & $\mathrm{C} 28$ & C29 & C30 & $0.2(10)$ \\
\hline $\mathrm{Zn} 5$ & $\mathrm{~N} 23$ & $\mathrm{C} 47$ & $\mathrm{C} 46$ & $-170.6(4)$ & $\mathrm{C} 28$ & $\mathrm{C} 29$ & C30 & N16 & $\begin{array}{l}- \\
177.9(6)\end{array}$ \\
\hline Zn5 & $\mathrm{N} 23$ & $\mathrm{C} 47$ & $\mathrm{C} 48$ & $8.1(10)$ & $\mathrm{C} 28$ & $\mathrm{C} 29$ & $\mathrm{C} 30$ & $\mathrm{C} 25$ & $0.2(9)$ \\
\hline $\mathrm{Zn} 54$ & N26 & C64 & $\mathrm{C} 1$ & $-9.5(9)$ & $\mathrm{C} 30$ & N16 & N17 & N18 & $-1.3(6)$ \\
\hline Zn54 & N26 & C64 & $\mathrm{C} 5$ & $170.5(4)$ & C30 & $\mathrm{C} 25$ & C26 & $\mathrm{C} 27$ & $0.2(8)$ \\
\hline N1 & $\mathrm{N} 25$ & N26 & Zn54 & $-170.8(4)$ & C31 & N19 & C37 & C32 & $0.7(7)$ \\
\hline N1 & N25 & N26 & C64 & $0.0(6)$ & C31 & N19 & C37 & C36 & $178.7(7)$ \\
\hline N1 & $\mathrm{C} 5$ & C64 & N26 & $0.1(6)$ & C31 & N20 & C32 & C33 & $\begin{array}{l}- \\
178.1(6)\end{array}$ \\
\hline N1 & $\mathrm{C} 5$ & C64 & $\mathrm{C} 1$ & $-179.9(5)$ & C31 & N20 & C32 & C37 & $0.7(6)$ \\
\hline N2 & N3 & N4 & Zn53 & $-159.7(4)$ & C32 & N20 & C31 & N19 & $-0.2(7)$ \\
\hline N2 & N3 & N4 & C6 & $-0.3(6)$ & C32 & C33 & C34 & C35 & $0.8(9)$ \\
\hline N3 & $\mathrm{N} 2$ & $\mathrm{C} 11$ & C6 & $-0.9(6)$ & C33 & C32 & C37 & N19 & $178.0(5)$ \\
\hline N3 & $\mathrm{N} 2$ & $\mathrm{C} 11$ & $\mathrm{C} 10$ & $179.8(6)$ & C33 & C32 & C37 & C36 & $-0.2(9)$ \\
\hline N3 & N4 & C6 & $\mathrm{C} 7$ & $178.9(6)$ & C33 & C34 & C35 & C36 & $-1.5(11)$ \\
\hline N3 & N4 & C6 & C11 & $-0.2(6)$ & C34 & $\mathrm{C} 35$ & C36 & C37 & $1.2(10)$ \\
\hline
\end{tabular}




\begin{tabular}{|c|c|c|c|c|c|c|c|c|c|}
\hline N4 & C6 & $\mathrm{C} 7$ & $\mathrm{C} 8$ & $178.9(6)$ & C35 & $\mathrm{C} 36$ & $\mathrm{C} 37$ & N19 & $\begin{array}{l}- \\
178.2(6)\end{array}$ \\
\hline N4 & C6 & $\mathrm{C} 11$ & N2 & $0.7(6)$ & C35 & $\mathrm{C} 36$ & $\mathrm{C} 37$ & C32 & $-0.4(9)$ \\
\hline N4 & C6 & $\mathrm{C} 11$ & $\mathrm{C} 10$ & $-179.9(5)$ & C37 & N19 & $\mathrm{C} 31$ & N20 & $-0.3(7)$ \\
\hline N5 & N6 & N7 & $\mathrm{Zn} 2$ & $176.4(4)$ & C37 & $\mathrm{C} 32$ & $\mathrm{C} 33$ & C34 & $0.0(9)$ \\
\hline N5 & N6 & N7 & C58 & $-0.4(7)$ & C38 & C39 & $\mathrm{C} 40$ & $\mathrm{C} 41$ & $-0.1(13)$ \\
\hline N6 & N5 & C63 & C58 & $1.5(6)$ & C39 & N8 & N9 & N10 & $0.0(7)$ \\
\hline N6 & N5 & C63 & C62 & $-177.6(6)$ & C39 & C38 & $\mathrm{C} 43$ & $\mathrm{C} 42$ & $0.1(12)$ \\
\hline N6 & N7 & C58 & C59 & $-178.3(7)$ & C39 & $\mathrm{C} 40$ & $\mathrm{C} 41$ & $\mathrm{C} 42$ & $2.2(16)$ \\
\hline N6 & N7 & C58 & C63 & $1.3(7)$ & $\mathrm{C} 40$ & $\mathrm{C} 41$ & $\mathrm{C} 42$ & $\mathrm{C} 43$ & $-3.3(18)$ \\
\hline N7 & $\mathrm{C} 58$ & C59 & C60 & $179.9(6)$ & $\mathrm{C} 41$ & $\mathrm{C} 42$ & $\mathrm{C} 43$ & C38 & $2.0(15)$ \\
\hline N7 & $\mathrm{C} 58$ & C63 & N5 & $-1.7(7)$ & $\mathrm{C} 43$ & C38 & C39 & N8 & $178.0(7)$ \\
\hline N7 & C58 & C63 & C62 & $177.5(5)$ & $\mathrm{C} 43$ & C38 & C39 & $\mathrm{C} 40$ & $-1.1(11)$ \\
\hline N8 & N9 & N10 & Zn3 & $170.8(4)$ & N44 & N21 & $\mathrm{C} 57$ & C52 & $0.5(7)$ \\
\hline N8 & N9 & N10 & C38 & $-0.7(7)$ & N44 & N21 & $\mathrm{C} 57$ & C56 & $\begin{array}{l}- \\
178.0(6)\end{array}$ \\
\hline N8 & C39 & $\mathrm{C} 40$ & C41 & $-178.9(8)$ & N44 & N22 & C52 & C53 & $\begin{array}{l}- \\
179.5(7)\end{array}$ \\
\hline N9 & N8 & C39 & C38 & $0.6(7)$ & N44 & N22 & $\mathrm{C} 52$ & $\mathrm{C} 57$ & $1.3(6)$ \\
\hline N9 & N8 & C39 & $\mathrm{C} 40$ & $179.6(8)$ & $\mathrm{C} 45$ & N23 & $\mathrm{C} 47$ & $\mathrm{C} 46$ & $1.6(6)$ \\
\hline N9 & N10 & C38 & C39 & $1.0(7)$ & $\mathrm{C} 45$ & N23 & $\mathrm{C} 47$ & $\mathrm{C} 48$ & $\begin{array}{l}- \\
179.7(6)\end{array}$ \\
\hline N9 & N10 & C38 & $\mathrm{C} 43$ & $-177.8(7)$ & $\mathrm{C} 45$ & N24 & $\mathrm{C} 46$ & $\mathrm{C} 47$ & $1.8(6)$ \\
\hline N10 & $\mathrm{C} 38$ & C39 & N8 & $-1.0(7)$ & $\mathrm{C} 45$ & N24 & $\mathrm{C} 46$ & C51 & $\begin{array}{l}- \\
177.5(6)\end{array}$ \\
\hline N10 & C38 & C39 & $\mathrm{C} 40$ & $179.9(7)$ & $\mathrm{C} 46$ & N24 & $\mathrm{C} 45$ & N23 & $-0.9(6)$ \\
\hline N10 & $\mathrm{C} 38$ & $\mathrm{C} 43$ & $\mathrm{C} 42$ & $178.8(8)$ & $\mathrm{C} 46$ & $\mathrm{C} 47$ & $\mathrm{C} 48$ & C49 & $1.7(8)$ \\
\hline N11 & N12 & N13 & Zn4 & $169.6(4)$ & $\mathrm{C} 47$ & N23 & $\mathrm{C} 45$ & N24 & $-0.5(7)$ \\
\hline N11 & N12 & N13 & C19 & $0.4(6)$ & $\mathrm{C} 47$ & $\mathrm{C} 46$ & $\mathrm{C} 51$ & $\mathrm{C} 50$ & $0.0(9)$ \\
\hline N12 & N11 & $\mathrm{C} 24$ & C19 & $0.7(6)$ & $\mathrm{C} 47$ & C48 & C49 & C50 & $-0.3(9)$ \\
\hline N12 & N11 & $\mathrm{C} 24$ & $\mathrm{C} 23$ & $-177.5(6)$ & C48 & C49 & $\mathrm{C} 50$ & C51 & $-1.3(11)$ \\
\hline N12 & N13 & C19 & $\mathrm{C} 20$ & $176.3(6)$ & C49 & $\mathrm{C} 50$ & C51 & $\mathrm{C} 46$ & $1.4(10)$ \\
\hline N12 & N13 & C19 & $\mathrm{C} 24$ & $0.0(6)$ & C51 & $\mathrm{C} 46$ & $\mathrm{C} 47$ & N23 & $177.3(5)$ \\
\hline N13 & C19 & $\mathrm{C} 20$ & $\mathrm{C} 21$ & $-176.9(6)$ & C51 & $\mathrm{C} 46$ & $\mathrm{C} 47$ & $\mathrm{C} 48$ & $-1.6(9)$ \\
\hline N13 & C19 & $\mathrm{C} 24$ & N11 & $-0.4(6)$ & C52 & N22 & N44 & N21 & $-1.1(7)$ \\
\hline N13 & C19 & $\mathrm{C} 24$ & $\mathrm{C} 23$ & $178.0(5)$ & C52 & $\mathrm{C} 53$ & C54 & C55 & $0.2(12)$ \\
\hline N15 & $\mathrm{C} 13$ & $\mathrm{C} 14$ & $\mathrm{C} 15$ & $178.7(10)$ & C53 & C52 & $\mathrm{C} 57$ & N21 & 179.6(6) \\
\hline N15 & C13 & $\mathrm{C} 18$ & N14 & $-0.8(8)$ & C53 & C52 & $\mathrm{C} 57$ & C56 & $-1.7(9)$ \\
\hline N15 & $\mathrm{C} 13$ & $\mathrm{C} 18$ & $\mathrm{C} 17$ & $179.4(7)$ & C53 & $\mathrm{C} 54$ & C55 & C56 & $0.1(12)$ \\
\hline N16 & N17 & N18 & Zn41 & $170.7(4)$ & C54 & C55 & C56 & $\mathrm{C} 57$ & $-1.1(11)$ \\
\hline N16 & N17 & N18 & $\mathrm{C} 25$ & $0.4(6)$ & C55 & $\mathrm{C} 56$ & $\mathrm{C} 57$ & N21 & $\begin{array}{l}- \\
179.7(6)\end{array}$ \\
\hline N17 & N16 & C30 & $\mathrm{C} 25$ & $1.6(6)$ & C55 & C56 & C57 & C52 & $1.9(9)$ \\
\hline N17 & N16 & C30 & $\mathrm{C} 29$ & $179.9(6)$ & $\mathrm{C} 57$ & N21 & N44 & N22 & $0.3(7)$ \\
\hline
\end{tabular}




\begin{tabular}{|c|c|c|c|c|c|c|c|c|c|}
\hline N17 & N18 & $\mathrm{C} 25$ & $\mathrm{C} 26$ & $-178.8(6)$ & C57 & C52 & C53 & C54 & $0.6(10)$ \\
\hline N17 & N18 & $\mathrm{C} 25$ & $\mathrm{C} 30$ & $0.6(6)$ & C58 & C59 & C60 & C61 & $2.6(9)$ \\
\hline N18 & $\mathrm{C} 25$ & $\mathrm{C} 26$ & $\mathrm{C} 27$ & $179.5(6)$ & C59 & C58 & C63 & N5 & $178.0(6)$ \\
\hline N18 & $\mathrm{C} 25$ & C30 & N16 & $-1.4(6)$ & C59 & C58 & C63 & C62 & $-2.8(9)$ \\
\hline N18 & $\mathrm{C} 25$ & C30 & $\mathrm{C} 29$ & $-179.9(5)$ & C59 & C60 & C61 & C62 & $-3.2(10)$ \\
\hline N20 & C32 & C33 & C34 & $178.5(6)$ & C60 & C61 & C62 & C63 & $0.6(9)$ \\
\hline N20 & C32 & C37 & N19 & $-0.9(6)$ & C61 & C62 & C63 & N5 & $\begin{array}{l}- \\
178.7(6)\end{array}$ \\
\hline N20 & $\mathrm{C} 32$ & C37 & $\mathrm{C} 36$ & $-179.1(5)$ & C61 & C62 & C63 & C58 & $2.3(9)$ \\
\hline N22 & C52 & C53 & C54 & $-178.4(7)$ & C63 & N5 & N6 & N7 & $-0.7(7)$ \\
\hline N22 & C52 & C57 & N21 & $-1.2(6)$ & C63 & C58 & C59 & C60 & $0.3(9)$ \\
\hline N22 & C52 & C57 & C56 & $177.6(5)$ & C64 & $\mathrm{C} 1$ & $\mathrm{C} 2$ & $\mathrm{C} 3$ & $-1.0(9)$ \\
\hline N23 & $\mathrm{C} 47$ & $\mathrm{C} 48$ & C49 & $-176.9(6)$ & C2S_1 & N1S_1 & C4S_1 & O5S_1 & $166(2)$ \\
\hline N24 & $\mathrm{C} 46$ & C47 & N23 & $-2.2(6)$ & C3S_1 & N1S_1 & C4S_1 & O5S_1 & $-14(3)$ \\
\hline N24 & $\mathrm{C} 46$ & $\mathrm{C} 47$ & $\mathrm{C} 48$ & $179.0(5)$ & C2S_2 & N1S_2 & C4S_2 & O5S_2 & $166(4)$ \\
\hline N24 & $\mathrm{C} 46$ & C51 & $\mathrm{C} 50$ & $179.3(6)$ & C3S_2 & N1S_2 & C4S_2 & O5S_2 & $-7(4)$ \\
\hline
\end{tabular}

Table S11. Hydrogen Atom Coordinates $\left(\AA \times 10^{4}\right)$ and Isotropic Displacement Parameters $\left(\AA^{2} \times 10^{3}\right)$ for GT-18.

$\begin{array}{lllll}\text { Atom } & \boldsymbol{x} & \boldsymbol{y} & \boldsymbol{z} & \mathbf{U}(\mathbf{e q}) \\ \text { H1 } & 5593.88 & -3726.39 & -751.65 & 30 \\ \text { H2 } & 7523.57 & -4442.28 & -973.51 & 34 \\ \text { H3 } & 9619.84 & -3783.62 & -910.51 & 34 \\ \text { H4 } & 9832.87 & -2391.07 & -602.86 & 28 \\ \text { H7 } & 14755.92 & -88.79 & -720.52 & 27 \\ \text { H8 } & 14386.26 & 1266.97 & -1079.08 & 32 \\ \text { H9 } & 12278.34 & 1899.82 & -1089.68 & 33 \\ \text { H10 } & 10470.55 & 1194.27 & -718.76 & 29 \\ \text { H12 } & 7704.03 & -133.2 & 3843.7 & 20 \\ \text { H14 } & 4882.18 & 186.56 & 1924.83 & 80 \\ \text { H15 } & 2859.1 & -503.21 & 1813.9 & 100 \\ \text { H16 } & 2068.15 & -1263.72 & 2640.13 & 90 \\ \text { H17 } & 3358.37 & -1430.76 & 3588.75 & 54 \\ \text { H20 } & 5449.77 & 1.96 & 5820.5 & 29 \\ \text { H21 } & 5772.51 & 1130.85 & 6495.66 & 38 \\ \text { H22 } & 7813.44 & 1807.3 & 6634.88 & 34 \\ \text { H23 } & 9666.27 & 1354.05 & 6130.01 & 32 \\ \text { H26 } & 14584.86 & -3118.57 & 5200.04 & 28 \\ \text { H27 } & 12732.1 & -3873 & 5500.69 & 33 \\ \text { H28 } & 10692.26 & -3231.75 & 5584.7 & 37\end{array}$




$\begin{array}{lllll}\text { H29 } & 10379.78 & -1807.82 & 5388.44 & 35 \\ \text { H31 } & 12371.44 & 2098.66 & 4729.41 & 21 \\ \text { H33 } & 13807.26 & 2840.3 & 6870.91 & 29 \\ \text { H34 } & 13572.22 & 1866.85 & 7673.53 & 34 \\ \text { H35 } & 12735.42 & 510.17 & 7441.06 & 41 \\ \text { H36 } & 12042.1 & 138.13 & 6425.73 & 34 \\ \text { H40 } & 10838.38 & 1615.74 & 2159.95 & 69 \\ \text { H41 } & 13073.5 & 2025.99 & 2237.53 & 96 \\ \text { H42 } & 14511.84 & 1694.26 & 3112.08 & 97 \\ \text { H43 } & 13715.82 & 1051.75 & 4000.11 & 60 \\ \text { H45 } & 7767.63 & 1178.78 & 612.82 & 18 \\ \text { H48 } & 6174.05 & 2850.74 & -1170.91 & 30 \\ \text { H49 } & 6163.48 & 2280.22 & -2171.31 & 38 \\ \text { H50 } & 6911.67 & 932.09 & -2332.71 & 41 \\ \text { H51 } & 7782.44 & 100.78 & -1506.9 & 35 \\ \text { H53 } & 5316.08 & 4040.86 & 1659.55 & 38 \\ \text { H54 } & 5041.32 & 4466.61 & 2686.75 & 47 \\ \text { H55 } & 5854.84 & 3660.62 & 3530.94 & 40 \\ \text { H56 } & 6991.19 & 2404.85 & 3391.94 & 33 \\ \text { H59 } & 9014.02 & -1287.02 & 2885.72 & 32 \\ \text { H60 } & 9850.41 & -2635.14 & 2707.96 & 36 \\ \text { H61 } & 10498.86 & -3022.6 & 1731.2 & 35 \\ \text { H62 } & 10176.53 & -2154.49 & 859.73 & 32 \\ \text { H2SA_1 } & -539.05 & 4444.26 & 2262.06 & 201 \\ \text { H2SB_1 } & -928.65 & 3485.23 & 2242.48 & 201 \\ \text { H2SC_1 } & 257.22 & 3811.52 & 1859.25 & 201 \\ \text { H3SA_1 } & 2253.1 & 4125.24 & 2308.19 & 144 \\ \text { H3SB_1 } & 2714.12 & 3616.79 & 2920.17 & 144 \\ \text { H3SC_1 } & 2199.52 & 4547.41 & 2975.09 & 144 \\ \text { H4S_1 } & -320.6 & 3252.52 & 3429.01 & 118 \\ \text { H2SA_2 } & 1368.27 & 4297.4 & 1313.46 & 201 \\ \text { H2SB_2 } & -191.29 & 4243.37 & 1364.49 & 201 \\ \text { H2SC_2 } & 642.67 & 3418.95 & 1271.39 & 201 \\ \text { H3SA_2 } & 2394.81 & 3084.5 & 2269.26 & 144 \\ \text { H3SB_2 } & 2254.89 & 3693.37 & 2844.2 & 144 \\ \text { H3SC_2 } & 2833.84 & 4031.69 & 2223.29 & 144 \\ \text { H4S_2 } & -870.59 & 4084.93 & 2474.19 & 118\end{array}$


Table S12. Atomic Occupancy for GT-18.

$\begin{array}{llllll}\text { Atom } & \text { Occupancy } & \text { Atom } & \text { Occupancy } & \text { Atom } & \text { Occupancy } \\ \text { O5S_1 } & 0.768(9) & \text { N1S_1 } & 0.768(9) & \text { C2S_1 } & 0.768(9) \\ \text { H2SA_1 } & 0.768(9) & \text { H2SB_1 } & 0.768(9) & \text { H2SC_1 } & 0.768(9) \\ \text { C3S_1 } & 0.768(9) & \text { H3SA_1 } & 0.768(9) & \text { H3SB_1 } & 0.768(9) \\ \text { H3SC_1 } & 0.768(9) & \text { C4S_1 } & 0.768(9) & \text { H4S_1 } & 0.768(9) \\ \text { O5S_2 } & 0.232(9) & \text { N1S_2 } & 0.232(9) & \text { C2S_2 } & 0.232(9) \\ \text { H2SA_2 } & 0.232(9) & \text { H2SB_2 } & 0.232(9) & \text { H2SC_2 } & 0.232(9) \\ \text { C3S_2 } & 0.232(9) & \text { H3SA_2 } & 0.232(9) & \text { H3SB_2 } & 0.232(9) \\ \text { H3SC_2 } & 0.232(9) & \text { C4S_2 } & 0.232(9) & \text { H4S_2 } & 0.232(9)\end{array}$




\section{Section 4: Tabular Data}

Table S13. The ratio of linkers ratio prepared from different BTA:BIM synthesis mole ratios at room temperature, as shown in Figure 1b. The final ratios were determined from $1 \mathrm{H}$ NMR after digestion of the MOF. The 4:1 synthesis were repeated three times to ensure reproducibility.

\begin{tabular}{|l|l|l|}
\hline $\begin{array}{l}\text { Linker Ratios in } \\
\text { Synthesis Solution } \\
\text { (BTA:BIM) }\end{array}$ & $\begin{array}{l}\text { Percentage of BTA } \\
(\%)\end{array}$ & $\begin{array}{l}\text { Percentage of BIM } \\
(\%)\end{array}$ \\
\hline $1: 1$ & 20 & 80 \\
\hline $1: 4$ & 46 & 54 \\
\hline $4: 1$ & 68 & 32 \\
\cline { 2 - 3 } & 68 & 32 \\
\cline { 2 - 3 } & 71 & 29 \\
\hline
\end{tabular}

Table S14. Ethane and ethylene adsorption/desorption isotherms at $298 \mathrm{~K}$ for ZIF-7M 20 , as shown in Figure 4a.

\begin{tabular}{|l|l|l|l|}
\hline $\begin{array}{l}\text { Pressure } \\
\text { (Bar) }\end{array}$ & $\begin{array}{l}\text { Quantity } \\
\text { Adsorbed } \\
\text { (mmol/g) }\end{array}$ & $\begin{array}{l}\text { Pressure } \\
\text { (Bar) }\end{array}$ & $\begin{array}{l}\text { Quantity } \\
\text { Adsorbed } \\
\text { (mmol/g) }\end{array}$ \\
\hline Ethane & \multicolumn{2}{|l|}{} \\
\hline 0.001 & 0.01 & 0.001 & 0.01 \\
\hline 0.002 & 0.02 & 0.002 & 0.01 \\
\hline 0.003 & 0.03 & 0.003 & 0.01 \\
\hline 0.004 & 0.04 & 0.004 & 0.02 \\
\hline 0.005 & 0.05 & 0.005 & 0.03 \\
\hline 0.008 & 0.07 & 0.008 & 0.04 \\
\hline 0.011 & 0.09 & 0.011 & 0.05 \\
\hline 0.015 & 0.12 & 0.015 & 0.07 \\
\hline 0.022 & 0.16 & 0.022 & 0.09 \\
\hline 0.030 & 0.21 & 0.031 & 0.11 \\
\hline 0.043 & 0.27 & 0.043 & 0.15 \\
\hline 0.061 & 0.36 & 0.061 & 0.19 \\
\hline 0.087 & 0.47 & 0.087 & 0.24 \\
\hline 0.12 & 0.63 & 0.12 & 0.30 \\
\hline 0.17 & 0.85 & 0.17 & 0.37 \\
\hline 0.25 & 1.51 & 0.25 & 0.46 \\
\hline 0.37 & 1.97 & 0.35 & 0.60 \\
\hline 0.51 & 2.09 & 0.50 & 0.77 \\
\hline 0.72 & 2.21 & 0.70 & 1.11 \\
\hline 1.00 & 2.31 & 1.01 & 1.90 \\
\hline 0.65 & 2.19 & 0.66 & 1.62 \\
\hline & & & \\
\hline & & & \\
\hline
\end{tabular}




\begin{tabular}{|l|l|l|l|}
\hline 0.43 & 2.06 & 0.45 & 0.91 \\
\hline 0.28 & 1.91 & 0.29 & 0.58 \\
\hline 0.20 & 1.77 & 0.18 & 0.41 \\
\hline 0.14 & 1.42 & 0.13 & 0.32 \\
\hline 0.08 & 0.60 & 0.09 & 0.22 \\
\hline 0.06 & 0.39 & 0.06 & 0.16 \\
\hline 0.04 & 0.27 & 0.04 & 0.11 \\
\hline 0.03 & 0.20 & 0.03 & 0.07 \\
\hline 0.001 & 0.01 & 0.001 & 0.01 \\
\hline 0.002 & 0.02 & 0.002 & 0.01 \\
\hline 0.003 & 0.03 & 0.003 & 0.01 \\
\hline 0.004 & 0.04 & 0.004 & 0.02 \\
\hline 0.005 & 0.05 & 0.005 & 0.03 \\
\hline 0.008 & 0.07 & 0.008 & 0.04 \\
\hline 0.011 & 0.09 & 0.011 & 0.05 \\
\hline 0.015 & 0.12 & 0.015 & 0.07 \\
\hline
\end{tabular}

Table S15. Ethane and ethylene adsorption/desorption isotherms at $298 \mathrm{~K}$ for ZIF-7M 46 , as shown in Figure 4b.

\begin{tabular}{|l|l|l|l|}
\hline $\begin{array}{l}\text { Pressure } \\
\text { (Bar) }\end{array}$ & $\begin{array}{l}\text { Quantity } \\
\text { Adsorbed } \\
\text { (mmol/g) }\end{array}$ & $\begin{array}{l}\text { Pressure } \\
\text { (Bar) }\end{array}$ & $\begin{array}{l}\text { Quantity } \\
\text { Adsorbed } \\
\text { (mmol/g) }\end{array}$ \\
\hline Ethane & \multicolumn{1}{|l|}{} \\
\hline 0.001 & 0.001 & 0.001 & 0.001 \\
\hline 0.002 & 0.001 & 0.002 & 0.002 \\
\hline 0.003 & 0.001 & 0.003 & 0.002 \\
\hline 0.004 & 0.001 & 0.004 & 0.004 \\
\hline 0.006 & 0.00 & 0.005 & 0.01 \\
\hline 0.008 & 0.01 & 0.008 & 0.01 \\
\hline 0.011 & 0.01 & 0.011 & 0.01 \\
\hline 0.015 & 0.01 & 0.015 & 0.02 \\
\hline 0.022 & 0.02 & 0.022 & 0.03 \\
\hline 0.03 & 0.03 & 0.03 & 0.04 \\
\hline 0.06 & 0.05 & 0.06 & 0.06 \\
\hline 0.09 & 0.06 & 0.09 & 0.08 \\
\hline 0.12 & 0.09 & 0.12 & 0.10 \\
\hline 0.17 & 0.19 & 0.17 & 0.12 \\
\hline 0.26 & 0.81 & 0.25 & 0.15 \\
\hline 0.40 & 0.97 & 0.35 & 0.22 \\
\hline 0.52 & 1.00 & 0.50 & 0.32 \\
\hline 0.72 & 1.04 & 0.71 & 0.60 \\
\hline
\end{tabular}




\begin{tabular}{|l|l|l|l|}
\hline 1.00 & 1.07 & 1.00 & 1.07 \\
\hline 0.65 & 1.03 & 0.65 & 0.99 \\
\hline 0.43 & 0.99 & 0.45 & 0.65 \\
\hline 0.08 & 0.17 & 0.08 & 0.11 \\
\hline & & 0.04 & 0.07 \\
\hline
\end{tabular}

Table S16. Ethane and ethylene adsorption/desorption isotherms at $298 \mathrm{~K}$ for ZIF-7M 46 , as shown in Figure 4c.

\begin{tabular}{|l|l|l|l|}
\hline $\begin{array}{l}\text { Pressure } \\
\text { (Bar) }\end{array}$ & $\begin{array}{l}\text { Quantity } \\
\text { Adsorbed } \\
\text { (mmol/g) }\end{array}$ & $\begin{array}{l}\text { Pressure } \\
\text { (Bar) }\end{array}$ & $\begin{array}{l}\text { Quantity } \\
\text { Adsorbed } \\
\text { (mmol/g) }\end{array}$ \\
\hline Ethane & \multicolumn{1}{|l|}{ Ethylene } \\
\hline 0.001 & 0.003 & 0.001 & 0.01 \\
\hline 0.002 & 0.005 & 0.002 & 0.02 \\
\hline 0.003 & 0.007 & 0.003 & 0.02 \\
\hline 0.004 & 0.010 & 0.004 & 0.03 \\
\hline 0.005 & 0.02 & 0.005 & 0.05 \\
\hline 0.008 & 0.03 & 0.008 & 0.08 \\
\hline 0.011 & 0.04 & 0.012 & 0.11 \\
\hline 0.016 & 0.05 & 0.016 & 0.15 \\
\hline 0.022 & 0.06 & 0.022 & 0.19 \\
\hline 0.03 & 0.09 & 0.03 & 0.24 \\
\hline 0.06 & 0.11 & 0.06 & 0.32 \\
\hline 0.10 & 0.13 & 0.09 & 0.37 \\
\hline 0.12 & 0.14 & 0.12 & 0.40 \\
\hline 0.19 & 0.15 & 0.17 & 0.44 \\
\hline 0.25 & 0.15 & 0.25 & 0.46 \\
\hline 0.35 & 0.16 & 0.37 & 0.49 \\
\hline 0.50 & 0.17 & 0.50 & 0.51 \\
\hline 0.71 & 0.18 & 0.71 & 0.54 \\
\hline 1.00 & 0.19 & 1.00 & 0.57 \\
\hline 0.65 & 0.19 & 0.65 & 0.55 \\
\hline 0.43 & 0.19 & 0.43 & 0.53 \\
\hline 0.09 & 0.16 & 0.09 & 0.46 \\
\hline 0.04 & 0.13 & & \\
\hline & & & \\
\hline
\end{tabular}




\section{References}

1. Gándara, F.; Uribe- Romo, F. J.; Britt, D. K.; Furukawa, H.; Lei, L.; Cheng, R.; Duan, X.; O'Keeffe, M.; Yaghi, O. M., Porous, conductive metal- triazolates and their structural elucidation by the charge- flipping method. Chemistry-A European Journal 2012, 18 (34), 10595-10601.

2. Cai, W.; Lee, T.; Lee, M.; Cho, W.; Han, D.-Y.; Choi, N.; Yip, A. C.; Choi, J., Thermal structural transitions and carbon dioxide adsorption properties of zeolitic imidazolate framework7 (ZIF-7). Journal of the American Chemical Society 2014, 136 (22), 7961-7971.

3. Jiang, J.-Q.; Yang, C.-X.; Yan, X.-P., Postsynthetic ligand exchange for the synthesis of benzotriazole-containing zeolitic imidazolate framework. Chemical Communications 2015, 51 (30), 6540-6543.

4. Jayachandrababu, K. C.; Sholl, D. S.; Nair, S., Structural and Mechanistic Differences in Mixed-Linker Zeolitic Imidazolate Framework Synthesis by Solvent Assisted Linker Exchange and de Novo Routes. Journal of the American Chemical Society 2017, 139 (16), 5906-5915.

5. Kresse, G.; Furthmuller, J., Efficient iterative schemes for ab initio total-energy calculations using a plane-wave basis set. Phys. Rev. B 1996, 54 (16), 11169-11186.

6. $\quad$ Blochl, P. E., Projector Augmented-Wave Method. Phys. Rev. B 1994, 50 (24), $17953-$ 17979.

7. $\quad$ Perdew, J. P.; Burke, K.; Ernzerhof, M., Generalized gradient approximation made simple. Phys. Rev. Lett. 1996, 77 (18), 3865-3868.

8. Grimme, S.; Antony, J.; Ehrlich, S.; Krieg, H., A consistent and accurate ab initio parametrization of density functional dispersion correction (DFT-D) for the 94 elements H-Pu. $J$. Chem. Phys. 2010, 132 (15), 154104.

9. Gücüyener, C.; van den Bergh, J.; Gascon, J.; Kapteijn, F., Ethane/ethene separation turned on its head: selective ethane adsorption on the metal- organic Framework ZIF-7 through a gateopening mechanism. Journal of the American Chemical Society 2010, 132 (50), 17704-17706.

10. Dubbeldam, D.; Calero, S.; Ellis, D. E.; Snurr, R. Q., RASPA: molecular simulation software for adsorption and diffusion in flexible nanoporous materials. Mol Simulat 2016, 42 (2), 81-101.

11. Manz, T. A.; Sholl, D. S., Improved Atoms-in-Molecule Charge Partitioning Functional for Simultaneously Reproducing the Electrostatic Potential and Chemical States in Periodic and Nonperiodic Materials. Journal of Chemical Theory and Computation 2012, 8 (8), 2844-2867.

12. Mayo, S. L.; Olafson, B. D.; Goddard, W. A., DREIDING - A GENERIC FORCE-FIELD FOR MOLECULAR SIMULATIONS. Journal of Physical Chemistry 1990, 94 (26), 8897-8909.

13. Martin, M. G.; Siepmann, J. I., Transferable potentials for phase equilibria. 1. United-atom description of n-alkanes. Journal of Physical Chemistry B 1998, 102 (14), 2569-2577.

14. Wick, C. D.; Martin, M. G.; Siepmann, J. I., Transferable potentials for phase equilibria. 4. United-atom description of linear and branched alkenes and alkylbenzenes. Journal of Physical Chemistry B 2000, 104 (33), 8008-8016.

15. Wells, B. A.; Chaffee, A. L., Ewald Summation for Molecular Simulations. J Chem Theory Comput 2015, 11 (8), 3684-95.

16. Crank, J., The mathematics of diffusion. Oxford university press: 1979.

17. Zhang, C.; Lively, R. P.; Zhang, K.; Johnson, J. R.; Karvan, O.; Koros, W. J., Unexpected molecular sieving properties of zeolitic imidazolate framework-8. The journal of physical chemistry letters 2012, 3 (16), 2130-2134. 
18. Park, K. S.; Ni, Z.; Côté, A. P.; Choi, J. Y.; Huang, R.; Uribe-Romo, F. J.; Chae, H. K.; O'Keeffe, M.; Yaghi, O. M., Exceptional chemical and thermal stability of zeolitic imidazolate frameworks. Proceedings of the National Academy of Sciences 2006, 103 (27), 10186-10191.

19. Grillo, F.; Tee, D. W.; Francis, S. M.; Früchtl, H. A.; Richardson, N. V., Passivation of Copper: Benzotriazole Films on $\mathrm{Cu}(111)$. The Journal of Physical Chemistry C 2014, 118 (16), 8667-8675.

20. $\quad$ Breck, D. W., Zeolite molecular sieves: structure, chemistry, and use. Wiley: 1973.

21. Koros, W. J.; Lively, R. P., Water and beyond: Expanding the spectrum of large- scale energy efficient separation processes. AIChE journal 2012, 58 (9), 2624-2633.

22. Bereciartua, P. J.; Cantín, Á.; Corma, A.; Jordá, J. L.; Palomino, M.; Rey, F.; Valencia, S.; Corcoran, E. W.; Kortunov, P.; Ravikovitch, P. I.; Burton, A.; Yoon, C.; Wang, Y.; Paur, C.; Guzman, J.; Bishop, A. R.; Casty, G. L., Control of zeolite framework flexibility and pore topology for separation of ethane and ethylene. Science 2017, 358 (6366), 1068.

23. Binder, T.; Chmelik, C.; Kärger, J.; Martinez-Joaristi, A.; Gascon, J.; Kapteijn, F.; Ruthven, D., A diffusion study of small hydrocarbons in DDR zeolites by micro-imaging. Microporous and Mesoporous Materials 2013, 180, 219-228.

24. Bachman, J. E.; Smith, Z. P.; Li, T.; Xu, T.; Long, J. R., Enhanced ethylene separation and plasticization resistance in polymer membranes incorporating metal-organic framework nanocrystals. Nature materials 2016, 15 (8), 845.

25. Bux, H.; Chmelik, C.; Krishna, R.; Caro, J., Ethene/ethane separation by the MOF membrane ZIF-8: molecular correlation of permeation, adsorption, diffusion. Journal of membrane science 2011, 369 (1-2), 284-289.

26. Gelles, T.; Lawson, S.; Thakkar, H.; Rezaei, F., Diffusion Kinetics of Ethane, Ethylene, and their Binary Mixtures in Ethane-Selective Adsorbents. Separation and Purification Technology 2019, 115872.

27. Sheldrick, G., SHELXT - Integrated space-group and crystal-structure determination. Acta Crystallographica Section A 2015, 71 (1), 3-8.

28. Dolomanov, O. V.; Bourhis, L. J.; Gildea, R. J.; Howard, J. A. K.; Puschmann, H., OLEX2:

a complete structure solution, refinement and analysis program. Journal of Applied Crystallography 2009, 42 (2), 339-341.

29. Sheldrick, G., Crystal structure refinement with SHELXL. Acta Crystallographica Section C 2015, 71 (1), 3-8.

30. Blatov, V. A.; Shevchenko, A. P.; Proserpio, D. M., Applied Topological Analysis of Crystal Structures with the Program Package ToposPro. Crystal Growth \& Design 2014, 14 (7), 3576-3586. 